\title{
Some pre-computed universal numeric arrays for linear convex quadrilateral finite elements
}

\author{
H.T. Rathod ${ }^{\mathrm{a}, *}$, Md. Shafiqul Islam ${ }^{\mathrm{b}}$ \\ ${ }^{a}$ Department of Mathematics, Central College Campus, Bangalore University, Bangalore, 560001, India \\ ${ }^{\mathrm{b}}$ Department of Mathematics, Shahjalal University of Science and Technology, Sylhet, Bangladesh
}

\begin{abstract}
In this paper we investigate the finite element analysis for the solution of linear partial differential equations using linear convex quadrilateral elements. We show that a linear polygonal domain can be discretized into a set of special linear convex quadrilateral elements, which generate the same expression for the determinant of the Jacobian matrix under the isoparametric coordinate transformation. Analytical integration of the above element matrices is shown to depend on certain 'universal pre-computed numeric arrays', i.e., the arrays which are computed once, stored on a permanent file and then reused in all subsequent applications of the program. We have constructed such arrays for the five commonly used linear quadrilateral elements: $Q 4, Q 8, Q 9, Q 12$ and $Q 16$. One speciality of these pre-computed arrays is that the arrays for lower order elements are already contained in the arrays for higher order elements. The performance of the proposed method is demonstrated by means of a numerical example. (C) 2001 Elsevier Science B.V. All rights reserved.
\end{abstract}

Keywords: Pre-computed numeric array; Quadrilateral element; Analytical integration

\section{Introduction}

In recent years, we have been witnessing finite element method (FEM) gaining importance due to the most obvious reason that it can provide solutions to many complicated problems that would be intractable by other numerical techniques [1,2]. In FEM, various integrals are to be determined numerically in the evaluation of the stiffness matrix, mass matrix, body force vector, initial stress vector, the surface load vector, etc. Among various numerical integration schemes, the use of Gauss quadrature is attractive and it can evaluate exactly the $(2 n-1)$ th order polynomials with

\footnotetext{
* Corresponding author.
} 
$n$ Gauss integration points used in view of the accuracy and efficiency of calculations. However, the integrands in practical situations are not always simple polynomials but rational expressions for which the Gauss quadrature scheme cannot be evaluated exactly [3]. The integration points have to be increased in order to improve the integration accuracy and it is desirable to make these evaluations by using as few Gauss points as possible, from the point of view of the computational efficiency. Thus it is an important task to strike a proper balance between accuracy and efficiency. Therefore, analytical integration is essential to generate a smaller error as well as to save the computational costs of Gauss quadrature commonly applied for science and engineering problems.

In this aspect a large number of articles are available in the literature. Among these, the researchers [4-8] have attempted to obtain the element matrices for general quadrilateral elements using analytical integration formulae. But the introduction of the isoparametric concept [4-7] or the subparametric concept with no curved edges limits the analytical integration of a displacement based finite element stiffness matrix to linear elements. Very recently, the algebraic manipulation language MAPLE has been used to investigate the explicit calculation of contributions to stiffness matrices for an isoparametric eight-noded quadrilateral element [8]. Complications arise from two main sources, firstly the large number of integrations that need to be performed and secondly, in methods which use isoparametric elements, the presence of the determinant of the Jacobian matrix (which will be referred to simply as the Jacobian throughout this paper) in the denominator of the element matrices. The objective of this paper is to obtain such matrices together with the principles: the modification of the finite element model by the discretization method and some 'pre-computed universal numeric arrays' [9] which do not depend on the element size or shape.

We begin with a brief discretization method for a two-dimensional linear polygon into a set of linear triangles in which each of these triangles is further discretized into three linear quadrilateral elements. These quadrilateral elements are mapped into 2 -squares $(-1 \leqslant \xi, \eta \leqslant 1)$ in the local parametric two-dimensional $(\xi, \eta)$ space to obtain the same expression of the Jacobian, namely $c(4+\xi+\eta)$ where $c$ is some appropriate constant. Next we present some basic analytical integration formulae for rational integrands arising in the universal arrays. The universal arrays are computed for the five commonly used linear quadrilaterals in FEM. Finally an application example is considered to verify the effectiveness of the proposed derivations. The results are demonstrated for Saint Venant's torsion problem [10-12] for an equilateral triangular cross section to compute the Prandtl stress values and the torsional constant by using the Serendipity and Lagrangian basis functions up to order three. The monotonic convergence from below is observed for cases with known analytical solutions.

\section{Explicit form of the Jacobian}

Let us consider an arbitrary four noded linear convex quadrilateral element in the global system $(x, y)$ which is mapped into a 2 -square in the local parametric system $(\xi, \eta)$ as shown in Fig. 1 . Then the isoparametric coordinate transformation from $(x, y)$ plane to $(\xi, \eta)$ plane is given by

$$
x=\sum_{k=1}^{4} x_{k} M_{k}(\xi, \eta), \quad y=\sum_{k=1}^{4} y_{k} M_{k}(\xi, \eta),
$$




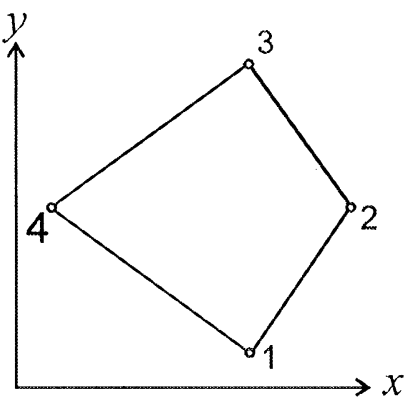

(a)

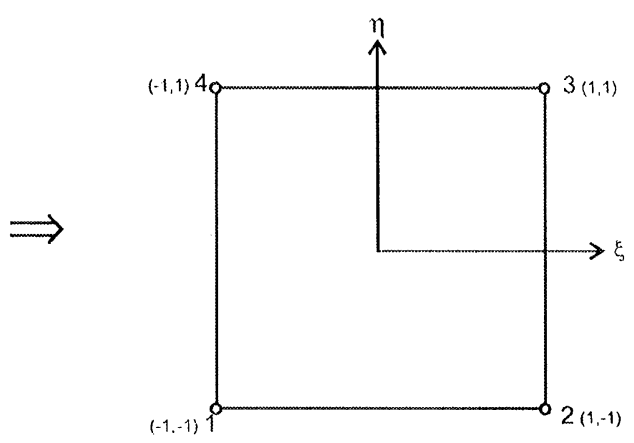

(b)

Fig. 1. Finite element geometry. (a) Original arbitrary Quadrilateral element in $(x, y)$ plane and (b) normalized 2-square in $(\xi, \eta)$ plane.

where $\left(x_{k}, y_{k}\right), k=1-4$, are the vertices of the element in $(x, y)$-plane and $M_{k}(\xi, \eta)$ denotes the 2-D bilinear basis functions $[1,2]$ with $\left(\xi_{k}, \eta_{k}\right)$ as the natural coordinates in $(\xi, \eta)$-plane such that

$$
M_{k}=\frac{1}{4}\left(1+\xi \xi_{k}\right)\left(1+\eta \eta_{k}\right), \quad k=1-4 .
$$

From Eqs. (1) and (2) we have

$$
\begin{aligned}
& \frac{\partial x}{\partial \xi}=\sum_{k=1}^{4} x_{k} \frac{\partial M_{k}}{\partial \xi}=\frac{1}{4}\left[\left(-x_{1}+x_{2}+x_{3}-x_{4}\right)+\left(x_{1}-x_{2}+x_{3}-x_{4}\right) \eta\right], \\
& \frac{\partial x}{\partial \xi}=\sum_{k=1}^{4} x_{k} \frac{\partial M_{k}}{\partial \xi}=\frac{1}{4}\left[\left(-x_{1}-x_{2}+x_{3}+x_{4}\right)+\left(x_{1}-x_{2}+x_{3}-x_{4}\right) \xi\right] .
\end{aligned}
$$

Similarly,

$$
\begin{aligned}
& \frac{\partial y}{\partial \xi}=\frac{1}{4}\left[\left(-y_{1}+y_{2}+y_{3}-y_{4}\right)+\left(y_{1}-y_{2}+y_{3}-y_{4}\right) \eta\right], \\
& \frac{\partial y}{\partial \eta}=\frac{1}{4}\left[\left(-y_{1}-y_{2}+y_{3}+y_{4}\right)+\left(y_{1}-y_{2}+y_{3}-y_{4}\right) \xi\right] .
\end{aligned}
$$

Hence the Jacobian, $J[1,2]$ can be expressed as

$$
J=\frac{\partial(x, y)}{\partial(\xi, \eta)}=\frac{\partial x}{\partial \xi} \frac{\partial y}{\partial \eta}-\frac{\partial x}{\partial \eta} \frac{\partial y}{\partial \xi}=\alpha_{0}+\alpha_{1} \xi+\alpha_{2} \eta
$$

where

$$
\begin{aligned}
& \alpha_{0}=\frac{1}{8}\left[\left(x_{4}-x_{2}\right)\left(y_{1}-y_{3}\right)+\left(x_{3}-x_{1}\right)\left(y_{4}-y_{2}\right)\right], \\
& \alpha_{1}=\frac{1}{8}\left[\left(x_{4}-x_{3}\right)\left(y_{2}-y_{1}\right)+\left(x_{1}-x_{2}\right)\left(y_{4}-y_{3}\right)\right], \\
& \alpha_{2}=\frac{1}{8}\left[\left(x_{4}-x_{1}\right)\left(y_{2}-y_{3}\right)+\left(x_{3}-x_{2}\right)\left(y_{4}-y_{1}\right)\right] .
\end{aligned}
$$




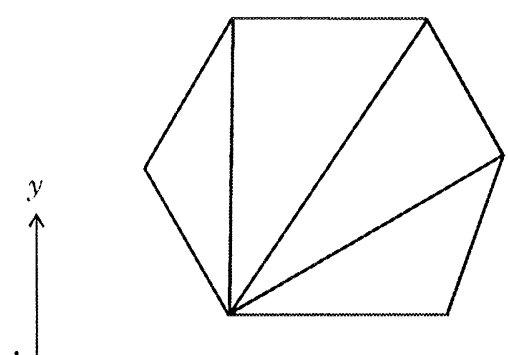

(a)

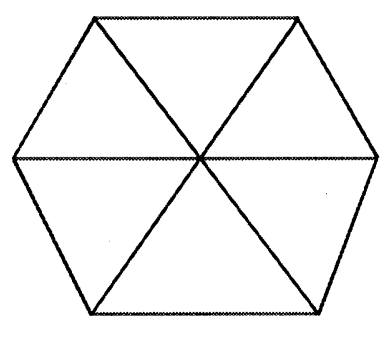

(b)

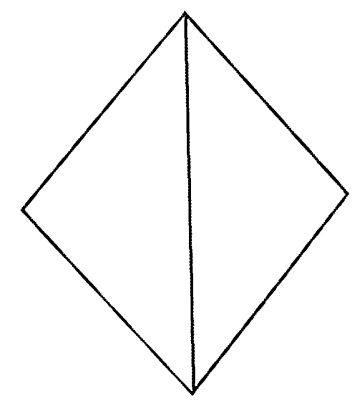

(c)

Fig. 2. Simple convex polygons which are decomposable into a set of triangles.

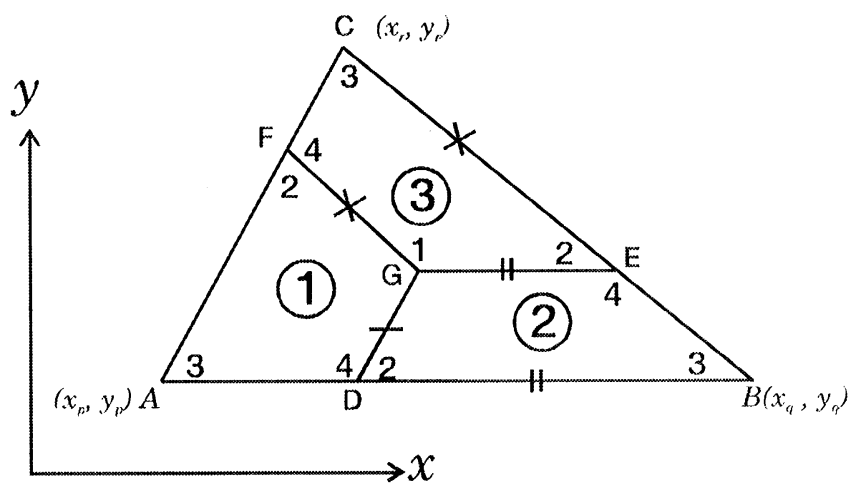

Fig. 3. An arbitrary triangle which is divided into three quadrilateral finite elements.

Without loss of generality let us consider some two-dimensional convex linear polygons in $(x, y)$ plane. Then we can construct a set of linear triangles as shown in Fig. 2 for each of the polygons. However, we would like to focus our attention on a particular dizcretization of a triangle and this will be the subject of the following Lemma.

Lemma 1. Let $A B C$ be an arbitrary triangle with vertices $A\left(x_{p}, y_{p}\right), B\left(x_{q}, y_{q}\right)$, and $C\left(x_{r}, y_{r}\right) ; D, E$ and $F$ be the mid-points of the sides $A B, B C$ and $C A$, respectively, and $G$ be its centroid. We can obtain three quadrilaterals GECF, GF AD and GDBE from this triangle as shown in Fig. 3. If we map each of these quadrilaterals into 2 squares in which the local nodes are oriented in counter clockwise from $G$, then the Jacobian for each element e is equivalent to

$$
J=J^{e}=\frac{1}{48} \Delta_{p q r}(4+\xi+\eta), e=(1),(2), \text { (3), }
$$

where $\Delta_{p q r}$ is the area of the triangle $A B C$. 
Proof. Since

$$
\Delta_{p q r}=\frac{1}{2}\left|\begin{array}{ccc}
1 & x_{p} & y_{p} \\
1 & x_{q} & y_{q} \\
1 & x_{r} & y_{r}
\end{array}\right|=\frac{1}{2}\left[\left(x_{p} y_{q}-x_{q} y_{p}\right)+\left(x_{q} y_{r}-x_{r} y_{q}\right)+\left(x_{r} y_{p}-x_{p} y_{r}\right)\right]
$$

Consider the quadrilateral GECF as element (1), the global and local nodes are then

$$
\begin{aligned}
& \left(x_{1}, y_{1}\right)=G:\left(\frac{x_{p}+x_{q}+x_{r}}{3}, \frac{y_{p}+y_{q}+y_{r}}{3}\right), \quad\left(x_{3}, y_{3}\right)=C:\left(x_{r}, y_{r}\right) \\
& \left(x_{2}, y_{2}\right)=E:\left(\frac{x_{q}+x_{r}}{2}, \frac{y_{q}+y_{r}}{3}\right), \quad\left(x_{4}, y_{4}\right)=F:\left(\frac{x_{p}+x_{r}}{2}, \frac{y_{p}+y_{r}}{2}\right) .
\end{aligned}
$$

Substituting Eq. (7) into Eq. (4b), we have

$$
\alpha_{0}=\frac{1}{12} \Delta_{p q r}, \quad \alpha_{1}=\frac{1}{48} \Delta_{p q r}, \quad \alpha_{0}=\frac{1}{48} \Delta_{p q r} .
$$

Hence using Eq. (8) in Eq. (4a) we have the Eq. (5). Similarly considering the other elements in Fig. 3, we can verify Eq. (5) easily.

\section{Global derivatives}

If $N_{i}^{e}$ denotes the basis functions of any order of the element $e$, then by the chain rule of partial differentiation from Eq. (1) we can write the global derivatives [1,2],

$$
\left\{\begin{array}{c}
\frac{\partial N_{i}^{e}}{\partial x} \\
\frac{\partial N_{i}^{e}}{\partial y}
\end{array}\right\}=\frac{1}{J}\left[\begin{array}{c}
\frac{\partial y}{\partial \eta}-\frac{\partial y}{\partial \xi} \\
-\frac{\partial x}{\partial \eta} \frac{\partial x}{\partial \xi}
\end{array}\right]\left\{\begin{array}{c}
\frac{\partial N_{i}^{e}}{\partial \xi} \\
\frac{\partial N_{i}^{e}}{\partial \eta}
\end{array}\right\}
$$

where $\partial x / \partial \xi, \partial x / \partial \eta, \partial y / \partial \xi$ and $\partial y / \partial \eta$ are defined in Eq. (3) while $J$ is defined in Eq. (5) and $i=1,2, \ldots$, tdof, (the total degrees of freedom) per element $e$.

\section{Element matrices}

In order to obtain the FE matrix using quadrilateral element due to a second order partial differential equation via Galerkin weighted residual formulation $[1,2]$ without numerical integration it suffices to develop the explicit formulas of the rational integrals of the form

$$
P_{i, j}^{x, x, e}=\int_{e} \frac{\partial N_{i}^{e}}{\partial x} \frac{\partial N_{j}^{e}}{\partial x} \mathrm{~d} x \mathrm{~d} y, \quad P_{i, j}^{y, y, e}=\int_{e} \frac{\partial N_{i}^{e}}{\partial y} \frac{\partial N_{j}^{e}}{\partial y} \mathrm{~d} x \mathrm{~d} y, \quad P_{i, j}^{x, y, e}=\int_{e} \frac{\partial N_{i}^{e}}{\partial x} \frac{\partial N_{j}^{e}}{\partial y} \mathrm{~d} x \mathrm{~d} y .
$$


In other words, the finite element matrix can be obtained by taking the linear combinations of the above equations in terms of the natural coordinates, which are given by

$$
\begin{aligned}
& P_{i, j}^{x, x}=\int_{-1}^{1} \int_{-1}^{1} \frac{1}{J}\left[\frac{\partial y}{\partial \eta} \frac{\partial N_{i}^{e}}{\partial \xi}-\frac{\partial y}{\partial \xi} \frac{\partial N_{i}^{e}}{\partial \eta}\right]\left[\frac{\partial y}{\partial \eta} \frac{\partial N_{j}^{e}}{\partial \xi}-\frac{\partial y}{\partial \xi} \frac{\partial N_{j}^{e}}{\partial \eta}\right] \mathrm{d} \xi \mathrm{d} \eta \\
& P_{i, j}^{y, y}=\int_{-1}^{1} \int_{-1}^{1} \frac{1}{J}\left[-\frac{\partial x}{\partial \eta} \frac{\partial N_{j}^{e}}{\partial \xi}+\frac{\partial x}{\partial \xi} \frac{\partial N_{i}^{e}}{\partial \eta}\right]\left[-\frac{\partial x}{\partial \eta} \frac{\partial N_{j}^{e}}{\partial \xi}+\frac{\partial x}{\partial \xi} \frac{\partial N_{j}^{e}}{\partial \eta}\right] \mathrm{d} \xi \mathrm{d} \eta \\
& P_{i, j}^{x, y}=\int_{-1}^{1} \int_{-1}^{1} \frac{1}{J}\left[\frac{\partial y}{\partial \eta} \frac{\partial N_{i}^{e}}{\partial \xi}-\frac{\partial y}{\partial \xi} \frac{\partial N_{i}^{e}}{\partial \eta}\right]\left[-\frac{\partial x}{\partial \eta} \frac{\partial N_{j}^{e}}{\partial \xi}+\frac{\partial x}{\partial \xi} \frac{\partial N_{j}^{e}}{\partial \eta}\right] \mathrm{d} \xi \mathrm{d} \eta .
\end{aligned}
$$

However, in this paper we shall develop those equations in an admissible matrix form to compute the element matrix easily.

\section{Some basic integration}

In this section, we introduce some basic integration of rational functions with linear denominator which will be employed in evaluating various integrals exactly.

Lemma 2. Let $\alpha$ be a positive real constant with $\alpha>|\beta|$ and $\beta \neq 0$, then the definite integral

$$
\begin{aligned}
& \int_{-1}^{1} x^{m} \ln (\alpha+\beta x) \mathrm{d} x=\frac{1}{m+1}\left[\left\{1-\left(-\frac{\alpha}{\beta}\right)^{m+1}\right\} \ln (\alpha+\beta)+\left\{(-1)^{m}+\left(-\frac{\alpha}{\beta}\right)^{m+1}\right\} \ln (\alpha-\beta)\right. \\
& \left.-\sum_{l=0}^{m}\left(\frac{\alpha}{\beta}\right)^{l} \frac{\left\{(-1)^{l}+(-1)^{m}\right\}}{m-l+1}\right]
\end{aligned}
$$

holds for all non-negative integer $m$.

Proof. Using integration by parts, we have

$$
\int x^{m} \ln (\alpha+\beta x) \mathrm{d} x==\frac{1}{m+1}\left[\left\{\ln (\alpha+\beta)+(-1)^{m} \ln (\alpha-\beta)\right\}-\int_{-1}^{1} \frac{\beta x^{m+1}}{\alpha+\beta x} \mathrm{~d} x\right] .
$$

Using the identity

$$
\frac{x^{m+1}}{\alpha+\beta x}=\frac{1}{\beta} \sum_{l=0}^{m}\left(-\frac{\alpha}{\beta}\right)^{l} x^{m-l}+\left(-\frac{\alpha}{\beta}\right)^{m+1} \frac{1}{\alpha+\beta x}, \quad m \geqslant 0
$$

in Eq. (13), the resulting Eq. (12) follows at once. 
Theorem 1. Let $m$ be any non-negative integer, then we have the explicit integral formula for the monomial of rational integrals

$$
I_{m, 0}=\int_{-1}^{1} \int_{-1}^{1} \frac{\xi^{m}}{4+\xi+\eta} \mathrm{d} \xi \mathrm{d} \eta=\int_{-1}^{1} \int_{-1}^{1} \frac{\eta^{m}}{4+\xi+\eta} \mathrm{d} \xi \mathrm{d} \eta=I_{0, m}
$$

and

$$
\begin{aligned}
I_{m, 0}= & {\left[\frac{1}{m+1}\left\{1-(-5)^{m+1}\right\} \ln \left(\frac{3}{2}\right)+\left\{(-1)^{m}+(-3)^{m+1}\right\} \ln 2\right.} \\
& \left.+\sum_{l=0}^{m} \frac{\left(3^{l}-5^{l}\right)\left\{(-1)^{m}+(-1)^{l}\right\}}{m-l+1}\right] .
\end{aligned}
$$

Proof. Using integration by parts, we have

$$
I_{m, 0}=\int_{-1}^{1} \int_{-1}^{1} \frac{\xi^{m}}{4+\xi+\eta} \mathrm{d} \xi \mathrm{d} \eta=\int_{-1}^{1} \xi^{m}[\ln (5+\xi)-\ln (3+\xi)] \mathrm{d} \xi .
$$

Hence using Lemma 2 in Eq. (16), we can get Eq. (15b) easily.

Now we quote the following simple results from Okabe [4, p. 205].

Lemma 3. With respect to the monomial $\xi^{m} \eta^{n}$, we have the explicit formula

$$
\int_{-1}^{1} \int_{-1}^{1} \xi^{m} \eta^{n} \mathrm{~d} \xi \mathrm{d} \eta=\frac{\left\{1+(-1)^{m}\right\}\left\{1+(-1)^{n}\right\}}{(m+1)(n+1)} .
$$

Theorem 2. For $m, n>0$, we have the rational integrals

$$
I_{m, n}=\int_{-1}^{1} \int_{-1}^{1} \frac{\xi^{m} \eta^{n}}{4+\xi+\eta} \mathrm{d} \xi \mathrm{d} \eta=\int_{-1}^{1} \int_{-1}^{1} \frac{\xi^{n} \eta^{m}}{4+\xi+\eta} \mathrm{d} \xi \mathrm{d} \eta=I_{n, m}
$$

and $I_{m, n}$ satisfies the following recurrence relation of the form:

$$
4 I_{m, n}+I_{m+1, n}+I_{m, n+1}=\frac{\left\{1+(-1)^{m}\right\}\left\{1+(-1)^{n}\right\}}{(m+1)(n+1)} .
$$

Proof. It follows easily from Okabe [4, p. 205]

\section{Explicit determination of the pre-computed arrays for the product of global derivatives}

In this section the pre-computed universal numeric arrays for the product of global derivatives for five quadrilateral elements commonly used in FEM will be given. For completeness and to facilitate the derivations, only the explicit expressions for the four node quadrilateral element will be given in detail. A brief account will also be given latter for the other elements. 
For this, let us write Eq. (1) in a parameterization form, namely

$$
t=\sum_{k=1}^{4} t_{k} M_{k}(\xi, \eta)=\frac{1}{4}\left(a_{t}+b_{t} \xi+c_{t} \eta+d_{t} \xi \eta\right)
$$

where

$$
\begin{array}{ll}
a_{t}=t_{1}+t_{2}+t_{3}+t_{4}, & c_{t}=-t_{1}-t_{2}+t_{3}+t_{4}, \\
b_{t}=-t_{1}+t_{2}+t_{3}-t_{4}, & d_{t}=t_{1}-t_{2}+t_{3}-t_{4},
\end{array} \quad t=x, y t_{k}=x_{k}, y_{k} .
$$

Then, Eq. (3) is equivalent to

$$
\frac{\partial t}{\partial \xi}=\frac{1}{4}\left(b_{t}+d_{t} \eta\right), \quad \frac{\partial t}{\partial \eta}=\frac{1}{4}\left(c_{t}+d_{t} \xi\right)
$$

The symbols $a_{t}, b_{t}, c_{t}$, and $d_{t}$ defined in Eq. (20) will be used throughout this paper.

Now, let us define

$$
\text { (i) } \phi=\left\{\begin{array}{ll}
x & \text { iff } t=y \\
y & \text { iff } t=x
\end{array} \phi \neq t \quad \text { (ii) } \Omega_{t}=\left\{\begin{aligned}
1 & \text { iff } t=y \\
-1 & \text { iff } t=x
\end{aligned}\right.\right.
$$

Then using Eqs. (9) and (21) we have

$$
\frac{\partial N_{i}^{e}}{\partial \phi}=\frac{\Omega_{t}}{J}\left[\frac{\partial t}{\partial \eta} \frac{\partial N_{i}^{e}}{\partial \xi}-\frac{\partial t}{\partial \xi} \frac{\partial N_{i}^{e}}{\partial \eta}\right]=\frac{\Omega_{t}}{4 J}\left[\left(c_{t}+d_{t} \xi\right) \frac{\partial N_{i}^{e}}{\partial \xi}-\left(b_{t}+d_{t} \eta\right) \frac{\partial N_{i}^{e}}{\partial \eta}\right] .
$$

Now we are ready to express the global derivatives and their products in explicit form.

\subsection{Four-noded quadrilateral (Q4) element}

Using the standard bilinear basis functions (Eq. (2)) in Eq. (23), we can write the global derivatives associated with the node $i$,

$$
\frac{\partial N_{i}^{e}}{\partial \phi}=\frac{\Omega_{t}}{16 J}\left[\begin{array}{lll}
a_{0,0}^{i, e}(t) & a_{1,0}^{i, e}(t) & a_{0,1}^{i, e}(t)
\end{array}\right]\left[\begin{array}{l}
1 \\
\xi \\
\eta
\end{array}\right], \quad 1 \leqslant i \leqslant 4,
$$

where $a_{\alpha, \beta}^{i, e}(t),(\alpha, \beta) \in\{(0,0),(1,0),(0,1)\}$ denotes the coefficients of the global derivatives corresponding to the polynomial $\xi^{\alpha} \eta^{\beta}$ which can be expressed as

$$
\begin{array}{llll}
a_{0,0}^{1, e}(t)=\left(b_{t}-c_{t}\right), & a_{0,0}^{2, e}(t)=\left(b_{t}+c_{t}\right), & a_{0,0}^{3, e}(t)=\left(-b_{t}+c_{t}\right), & a_{0,0}^{4, e}(t)=-\left(b_{t}+c_{t}\right), \\
a_{1,0}^{1, e}(t)=-\left(b_{t}+d_{t}\right), & a_{1,0}^{2, e}(t)=\left(b_{t}+d_{t}\right), & a_{1,0}^{3, e}(t)=\left(-b_{t}+d_{t}\right), & a_{1,0}^{4, e}(t)=\left(b_{t}-d_{t}\right), \\
a_{0,1}^{1, e}(t)=\left(c_{t}+d_{t}\right), & a_{0,1}^{2, e}(t)=\left(-c_{t}+d_{t}\right), & a_{0,1}^{3, e}(t)=\left(c_{t}-d_{t}\right), & a_{0,1}^{4, e}(t)=-\left(c_{t}+d_{t}\right) .
\end{array}
$$

Similarly, if $\psi$ and $s$ satisfy Eqs. (22)-(24) such that
(i) $\psi=\left\{\begin{array}{ll}x \text { iff } & s=y \\ y \text { iff } & s=x\end{array} \psi \neq s\right\}$
(ii) $\Omega_{s}=\left\{\begin{array}{rr}1 \text { iff } & s=y, \\ -1 \text { iff } & s=x\end{array}\right.$ 
and if we define

$$
\Omega_{t, s}=\left(\Omega_{t}\right)\left(\Omega_{s}\right)=\left\{\begin{aligned}
1 & \text { iff } t=s \\
-1 & \text { iff } t \neq s
\end{aligned}\right.
$$

then we can express the global derivatives associated with the node $j$, also in the form similar to

$$
\begin{aligned}
& \frac{\partial N_{j}^{e}}{\partial \psi}=\frac{\Omega_{s}}{16 J}\left[\begin{array}{lll}
1 & \xi & \eta
\end{array}\right]\left[\begin{array}{l}
a_{0,0}^{j, e}(s) \\
a_{1,0}^{j, e}(s) \\
a_{0,1}^{j, e}(s)
\end{array}\right], \quad 1 \leqslant j \leqslant 4, \\
& \frac{\partial N_{i}^{e}}{\partial \phi} \frac{\partial N_{j}^{e}}{\partial \psi}=\frac{\Omega_{t, s}}{256 J^{2}}\left[\begin{array}{lll}
a_{0,0}^{i, e}(t) & a_{1,0}^{i, e}(t) & a_{0,1}^{i, e}(t)
\end{array}\right]\left[\begin{array}{lll}
1 & \xi & \eta \\
\xi & \xi^{2} & \xi \eta \\
\eta & \xi \eta & \eta^{2}
\end{array}\right]\left[\begin{array}{l}
a_{0,0}^{j, e}(s) \\
a_{1,0}^{j, e}(s) \\
a_{0,1}^{j, e}(s)
\end{array}\right], \quad 1 \leqslant i, j \leqslant 4 .
\end{aligned}
$$

Thus the integrals that have to be evaluated are $P_{i, j}^{x, x, e}, P_{i, j}^{y, y, e}$ and $P_{i, j}^{x, y, e}$ in Eqs. (10) and (11) which can be written in symbolic form using Eqs. (27) and (5) such that

$$
\begin{aligned}
P_{i, j}^{\phi, \psi}, e & =\iint_{e}\left(\frac{\partial N_{i}^{e}}{\partial \phi} \frac{\partial N_{j}^{e}}{\partial \psi}\right) \mathrm{d} x \mathrm{~d} y \\
& =\frac{3 \Omega_{t, s}}{16 \Delta_{p q r}}\left[\begin{array}{lll}
a_{0,0}^{i, e}(t) & a_{1,0}^{i, e}(t) & a_{0,1}^{i, e}(t)
\end{array}\right]\left[\begin{array}{lll}
I_{0,0} & I_{1,0} & I_{0,1} \\
I_{1,0} & I_{2,0} & I_{1,1} \\
I_{0,1} & I_{1,1} & I_{0,2}
\end{array}\right]\left[\begin{array}{l}
a_{0,0}^{j, e}(s) \\
a_{1,0}^{j, e}(s) \\
a_{0,1}^{j, e}(s)
\end{array}\right], \quad 1 \leqslant i, j \leqslant 4,
\end{aligned}
$$

where the inner integrals are of the form

$$
I_{m, n}=\int_{-1}^{1} \int_{-1}^{1} \frac{\xi^{m} \eta^{n}}{4+\xi+\eta} \mathrm{d} \xi \mathrm{d} \eta, \quad m+n=0,1,2
$$

and are now to be evaluated analytically using Eqs. (15) and (19). Consequently, the final matrix form of Eq. (11) using Eq. (28) is given by

$$
P_{i, j}^{\phi, \psi, e}=\frac{3 \Omega_{t, s}}{16 \Delta_{p q r}}\left[a_{\alpha, \beta}^{i, e}(t)\right]_{1 \times 3}[S]_{3 \times 3}\left[a_{\alpha, \beta}^{j, e}(s)\right]_{3 \times 1},
$$

where $(\alpha, \beta) \in\{(0,0),(1,0),(0,1)\}$ and $[S]_{1 \times 3}$ is a symmetric matrix given by

$$
[S]_{3 \times 3}=\left[\begin{array}{rll}
1.046496288 & & \\
-0.092992575 & 0.355592309 & \text { symmetric } \\
-0.092992575 & 0.016377991 & 0.355592093
\end{array}\right]
$$

which is called the "pre-computed universal numeric array" for the $Q 4$ element. 


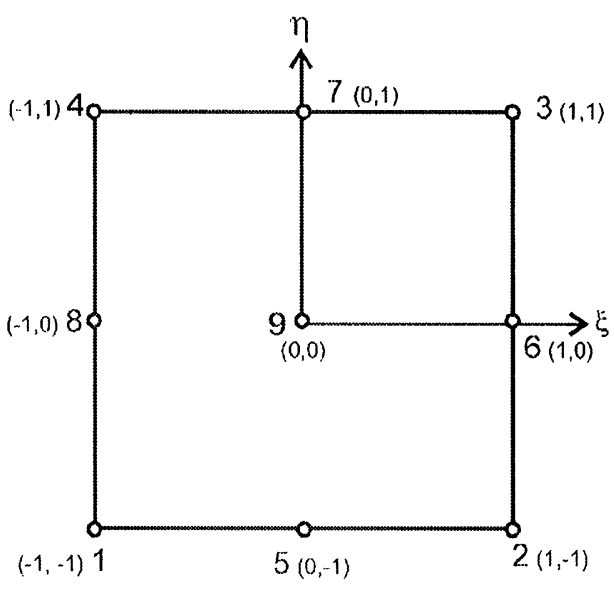

(a)

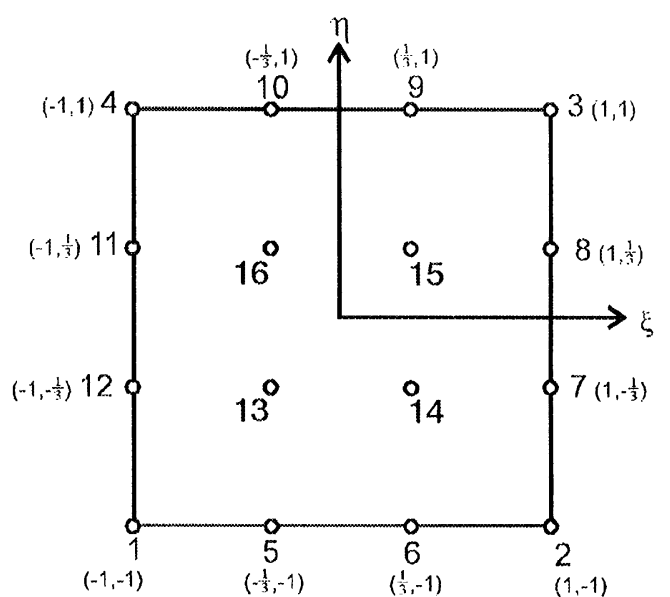

(b)

Fig. 4. Quadrilateral elements in $(\xi, \eta)$ plane: (a) $Q 8$ and $Q 9$, (b) $Q 12$ and $Q 16$.

\subsection{Eight-noded quadrilateral (Q8) element}

For the $Q 8$ elements shown in Fig. 4a we can write the matrix formulation for the product of global derivatives in a form similar to Eq. (30)

$$
P_{i, j}^{\phi, \psi, e}=\frac{3 \Omega_{t, s}}{16 \Delta_{p q r}}\left[a_{\alpha, \beta}^{i, e}(t)\right]_{1 \times 8}[S]_{8 \times 8}\left[a_{\alpha, \beta}^{j, e}(s)\right]_{8 \times 1}, \quad 1 \leqslant i, j \leqslant 8
$$

where $(\alpha, \beta) \in\{(0,0),(1,0),(0,1),(2,0),(1,1),(0,2),(2,1),(1,2)\}$, the matrix $[S]_{8 \times 8}$ can be obtained by taking first 8 rows and 8 columns from the matrix $[S]_{15 \times 15}$ which is given in Appendix B and the coefficient matrix $a_{\alpha, \beta}^{i, e}(t), i=1,2, \ldots, 8$ is given explicitly in Appendix A. The other variables have the same meaning as mentioned in earlier sections.

\subsection{Nine-noded quadrilateral $(Q 9)$ element}

For the $Q 9$ element shown in Fig. 4a, the Eq. (30) becomes

$$
P_{i, j}^{\phi, \psi, e}=\frac{3 \Omega_{t, s}}{16 \Delta_{p q r}}\left[a_{\alpha, \beta}^{i, e}(t)\right]_{1 \times 8}[S]_{8 \times 8}\left[a_{\alpha, \beta}^{j, e}(s)\right]_{8 \times 1}, \quad 1 \leqslant i, j \leqslant 9,
$$

where $(\alpha, \beta)$ and $[S]_{8 \times 8}$ are same as $Q 8$ elements in Eq. (31) while $a_{\alpha, \beta}^{i, e}(t), i=1,2, \ldots, 9$ is given explicitly in Appendix A.

\subsection{Twelve-noded quadrilateral (Q12) element}

Using $Q 12$ elements shown in Fig. 4b, the product of global derivatives can be written in a form similar to Eq. (30) such that

$$
P_{i, j}^{\phi, \psi, e}=\frac{3 \Omega_{t, s}}{1024 \Delta_{p q r}}\left[a_{\alpha, \beta}^{i, e}(t)\right]_{1 \times 12}[S]_{12 \times 12}\left[a_{\alpha, \beta}^{j, e}(s)\right]_{12 \times 1}, \quad 1 \leqslant i, j \leqslant 12
$$


where $(\alpha, \beta) \in\{(0,0),(1,0),(0,1),(2,0),(1,1),(0,2),(2,1),(1,2),(3,0),(0,3),(3,1),(1,3)\},[S]_{12 \times 12}$ is a submatrix obtained by taking first 12 rows and 12 columns from the matrix $[S]_{15 \times 15}$ given in Appendix B and $a_{\alpha, \beta}^{i, e}(t), i=1,2, \ldots, 12$ is given explicitly in Appendix A.

\subsection{Sixteen-noded quadrilateral (Q16) element}

For $Q 16$ elements shown in Fig. 4b, one can write the product of global derivatives in Eq. (11) using similar arguments described for $Q 4$ element as

$$
P_{i, j}^{\phi, \psi, e}=\frac{3 \Omega_{t, s}}{65336 \Delta_{p q r}}\left[a_{\alpha, \beta}^{i, e}(s)\right]_{1 \times 15}[S]_{15 \times 15}\left[a_{\alpha, \beta}^{j, e}(s)\right]_{15 \times 1}, \quad 1 \leqslant i, j \leqslant 16
$$

where $(\alpha, \beta) \in\{(0,0),(1,0),(0,1),(2,0),(1,1),(0,2),(2,1),(1,2),(3,0),(0,3),(3,1),(1,3),(2,2),(3,2),(2,3)\}$, $[S]_{15 \times 15}$ is a symmetric matrix given in Appendix B and the coefficients $a_{\alpha, \beta}^{i, e}(t), i=1,2, \ldots, 16$ of the global derivatives are given explicitly in Appendix A.

\section{Demonstrative example}

\subsection{Statement of the problem and its FE equation}

In this section we shall examine the application of the proposed derivations in detail considering the saint Venant Torsion Problem [11],

$$
\begin{aligned}
& \frac{\partial^{2} u}{\partial x^{2}}+\frac{\partial^{2} u}{\partial y^{2}}+2 G \theta=0 \text { in } R, \\
& u(x, y)=0 \text { on } \Gamma,
\end{aligned}
$$

where $u(x, y)$ is the Prandtl stress function, $G$ is the shear modulus and $\theta$ is the constant rate of twist along the axis of the bar in which $G \theta=1 . R$ refers to the interior and $\Gamma$ to the boundary of the equilateral triangular cross section with vertices $A\left(-\frac{1}{2}, 0\right), B\left(\frac{1}{2}, 0\right)$ and $C(0, \sqrt{315} 2)$ as shown in Fig. 5. Then the corresponding torsional constant $T$ is given by

$$
T=2 \iint_{R} u(x, y) \mathrm{d} x \mathrm{~d} y .
$$

Suppose that within each quadrilateral element $e$, the Prandtl stress function $u(x, y)$ is expressed in terms of the natural coordinate variables $\xi$ and $\eta$ such that

$$
u(x, y)=\sum_{i=1}^{m} N_{i}^{e}(\xi, \eta) u_{i}^{e},
$$

where $N_{i}(\xi, \eta)$ denotes the basis functions of quadrilateral element, $u_{i}$ is the nodal stress values and $m$ denotes the total degrees of freedom per element $e$. Using the coordinate transformation and Galerkin weighted residual method, the numerical solution of the above boundary value problem 


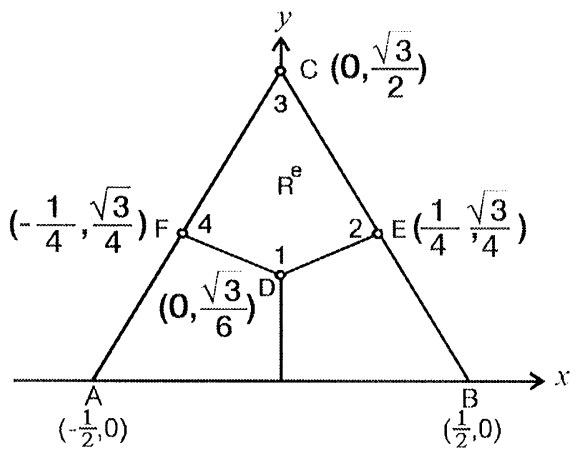

(a)

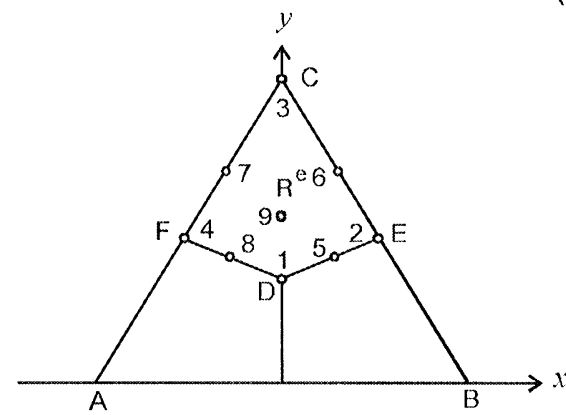

(b)

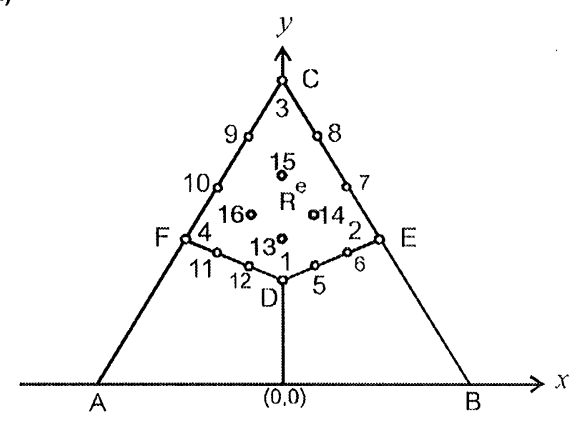

(c)

\section{Mesh 1}

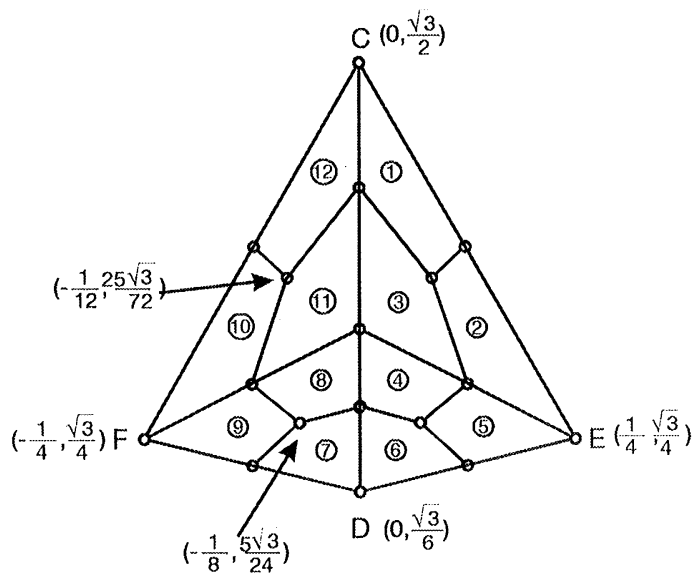

Mesh 2

Fig. 5. Equilateral triangular crosssection and its finite element meshes. 
in the domain $R^{e}$ is expressed [1,2] as

$$
\left[K_{i, j}^{e}\right]\left\{u_{i}^{e}\right\}=\left\{F_{i}^{e}\right\},
$$

where

$$
\begin{aligned}
& K_{i, j}^{e}=\iint_{R^{e}}\left(\frac{\partial N_{i}^{e}}{\partial x} \frac{\partial N_{j}^{e}}{\partial x}+\frac{\partial N_{i}^{e}}{\partial y} \frac{\partial N_{j}^{e}}{\partial y}\right) \mathrm{d} x \mathrm{~d} y=P_{i, j}^{x, x, e}+P_{i, j}^{y, y, e}, \\
& F_{i}^{e}=2 \iint_{R^{e}} N_{i}^{e}(\xi, \eta) \mathrm{d} x \mathrm{~d} y, \\
& T=\sum_{i=1}^{m} u_{i}^{e} F_{i}^{e} .
\end{aligned}
$$

\subsection{Discretization and finite element model}

In constructing a finite element model we shall discretize a particular triangle into three quadrilateral elements as described in Section 2 and also we can exploit if there are any symmetry. However, we shall consider the following two types of refined quadrilateral meshes:

Mesh 1: Due to the symmetry, only one third of the original model is considered to solve the above boundary value problem which can be redefined [10] as

$$
\begin{aligned}
& \frac{\partial^{2} u}{\partial x^{2}}+\frac{\partial^{2} u}{\partial y^{2}}=-2 \quad \text { within } R^{e}, \\
& u=0 \quad \text { on } \Gamma_{1}, \\
& \frac{\partial u}{\partial n}=0 \quad \text { on } \Gamma_{2},
\end{aligned}
$$

where $R^{e}$ is the quadrilateral element with vertices $G\left(0, \frac{\sqrt{3}}{6}\right), E\left(\frac{1}{4}, \frac{\sqrt{3}}{4}\right), C\left(0, \frac{\sqrt{3}}{2}\right)$ and $F\left(-\frac{1}{4}, \frac{\sqrt{3}}{4}\right)$; the boundary $\Gamma_{1}$ : sides $C E$ and $C F$ and the boundary $\Gamma_{2}$ : sides $G E$ and $G F$, are shown in Fig. 5.

Since only one element is considered, the improved solutions are obtained by increasing the polynomial level (i.e. $p$-version) of approximations. Following the proposed derivations of this paper using various basis functions the computed Prandtl Stress values at different locations are tabulated in Table 1 and compared with the exact solutions [12]. The torsional constants are also listed in Table 2 and compared with the existing solutions in the literature [10] obtained by hierarchical basis functions.

Mesh 2: Retaining the same boundary conditions the quadrilateral GECF obtained in mesh 1 is discretized into 4 triangles and each of the triangles is further discretized into three quadrilateral elements as shown in Fig. 5 (mesh 2). This refined mesh is considered only to show that the improved solutions can also be obtained by increasing the number of lowest order quadrilateral elements. The proposed derivation can be applied to obtain the element matrix easily. The computed torsional constant for this case is given by

$$
T=0.020449711 \text {. }
$$


Table 1

Prandtl stress function values at various points of Fig. 5

\begin{tabular}{|c|c|c|c|c|c|c|c|}
\hline \multirow{2}{*}{$\begin{array}{l}\text { Location of } \\
\text { points }\end{array}$} & \multicolumn{6}{|c|}{ Element (used) and corresponding stress values by the present method } & \multirow[t]{2}{*}{ Theoretical } \\
\hline & $Q 4$ & $Q 8$ & $Q 9$ & $Q 12$ & $Q 16$ & Q4 (Mesh 2) & \\
\hline$\left(0, \frac{\sqrt{3}}{6}\right)$ & 072064416 & 0.050110581 & 0.056084299 & 0.050698179 & 0.055555556 & 0.056769847 & 0.055555556 \\
\hline$\left(0, \frac{7 \sqrt{3}}{24}\right)$ & $0.01801604^{\mathrm{a}}$ & $0.035041736^{\mathrm{a}}$ & 0.038171991 & $0.034984252^{\mathrm{a}}$ & $0.037977431^{\mathrm{a}}$ & 0.037925442 & 0.037977431 \\
\hline$\left( \pm \frac{1}{12}, \frac{7 \sqrt{3}}{36}\right)$ & $0.048042944^{\mathrm{a}}$ & $0.053419578^{\mathrm{a}}$ & $0.050719977^{\mathrm{a}}$ & 0.052581625 & 0.050411523 & $0.045683308^{\mathrm{a}}$ & 0.050411523 \\
\hline$\left( \pm \frac{1}{8}, \frac{5 \sqrt{3}}{24}\right)$ & $0.036032208^{\mathrm{a}}$ & 0.047569381 & 0.043038900 & $0.047658796^{\mathrm{a}}$ & $0.043042778^{\mathrm{a}}$ & 0.044678662 & 0.043402778 \\
\hline$\left( \pm \frac{1}{6}, \frac{2 \sqrt{3}}{9}\right)$ & $0.024021472^{\mathrm{a}}$ & $0.036716052^{\mathrm{a}}$ & $0.032025211^{\mathrm{a}}$ & 0.037778257 & 0.032921811 & $0.044361587^{\mathrm{a}}$ & 0.032921811 \\
\hline$\left(0, \frac{13 \sqrt{3}}{54}\right)$ & $0.032028629^{\mathrm{a}}$ & $0.048954736^{\mathrm{a}}$ & $0.049933202^{\mathrm{a}}$ & $0.047576309^{\mathrm{a}}$ & 0.048544429 & $0.041506203^{\mathrm{a}}$ & 0.048544429 \\
\hline$\left(0, \frac{19 \sqrt{3}}{54}\right)$ & $0.008007157^{\mathrm{a}}$ & $0.018909525^{\mathrm{a}}$ & $0.022351473^{\mathrm{a}}$ & $0.019552373^{\mathrm{a}}$ & 0.023167200 & $0.028175029^{\mathrm{a}}$ & 0.023167200 \\
\hline$\left( \pm \frac{1}{12}, \frac{31 \sqrt{3}}{108}\right)$ & $0.016014314^{\mathrm{a}}$ & $0.03148209^{\mathrm{a}}$ & $0.033026543^{\mathrm{a}}$ & $0.03144645^{\mathrm{a}}$ & 0.033074226 & $0.020811340^{\mathrm{a}}$ & 0.033074226 \\
\hline
\end{tabular}

${ }^{\mathrm{a}}$ Obtained via the interpolating polynomial for the element.

Table 2

Torsional constant, $T$ for an equilateral triangular cross section of Fig. 5

\begin{tabular}{|c|c|c|c|c|c|c|c|c|c|}
\hline \multicolumn{6}{|c|}{ Element Mesh 1} & \multirow{2}{*}{$\begin{array}{l}\text { Mesh } 2 \\
Q 4\end{array}$} & \multicolumn{2}{|l|}{ Hierarchical [10] } & \multirow[t]{2}{*}{ Theoretical } \\
\hline & $Q 4$ & $Q 8$ & $Q 9$ & $Q 12$ & $Q 16$ & & $(p=1) \quad(p=2)$ & $(p=3)$ & \\
\hline $\mathrm{T}$ & 0.013002003 & 0.020956354 & 0.021579124 & 0.021056308 & 0.021650635 & 0.020449711 & $0.01313 \quad 0.02158$ & 0.02165 & 0.021650635 \\
\hline
\end{tabular}

Observe that the above torsional constant is also converging to the exact solution.

\section{Application to the computation of element stiffness matrix in plane elasticity}

From the standard two-dimensional strain-displacement relationship [1,2], the coefficient $K_{i, j}^{e}$, of the element matrix $\left[K^{e}\right]$ is of the form

$$
K_{i, j}^{e}=t \int_{e} \mathrm{~B}_{i}^{t} D \mathrm{~B}_{j} \mathrm{~d} x \mathrm{~d} y, \quad 1 \leqslant i, j \leqslant N,
$$


where

$$
\begin{gathered}
\mathrm{B}_{i}=\left[\begin{array}{cc}
\frac{\partial N_{i}^{e}}{\partial x} & 0 \\
0 & \frac{\partial N_{i}^{e}}{\partial y} \\
\frac{\partial N_{i}^{e}}{\partial y} & \frac{\partial N_{i}^{e}}{\partial x}
\end{array}\right], \\
D=\left[\begin{array}{ccc}
E_{x} & E_{x y} & 0 \\
E_{x y} & E_{y} & 0 \\
0 & 0 & G
\end{array}\right]
\end{gathered}
$$

and $t$ is the element thickness.

Then the integrand of Eq. (42) is a $2 \times 2$ matrix, which is given by

$$
K_{i, j}^{e}=\left[\begin{array}{ll}
S_{1,1}^{e} & S_{1,2}^{e} \\
S_{2,1}^{e} & S_{2,2}^{e}
\end{array}\right],
$$

where

$$
\begin{aligned}
& S_{1,1}^{e}=t \int_{e}\left(E_{x} \frac{\partial N_{i}^{e}}{\partial x} \frac{\partial N_{j}^{e}}{\partial x}+G \frac{\partial N_{i}^{e}}{\partial y} \frac{\partial N_{j}^{e}}{\partial y}\right) \mathrm{d} x \mathrm{~d} y, \\
& S_{1,2}^{e}=t \int_{e}\left(E_{x y} \frac{\partial N_{i}^{e}}{\partial x} \frac{\partial N_{j}^{e}}{\partial y}+G \frac{\partial N_{i}^{e}}{\partial y} \frac{\partial N_{j}^{e}}{\partial x}\right) \mathrm{d} x \mathrm{~d} y, \\
& S_{2,1}^{e}=t \int_{e}\left(E_{x y} \frac{\partial N_{i}^{e}}{\partial y} \frac{\partial N_{j}^{e}}{\partial x}+G \frac{\partial N_{i}^{e}}{\partial x} \frac{\partial N_{j}^{e}}{\partial y}\right) \mathrm{d} x \mathrm{~d} y, \\
& S_{2,2}^{e}=t \int_{e}\left(E_{y} \frac{\partial N_{i}^{e}}{\partial y} \frac{\partial N_{j}^{e}}{\partial y}+G \frac{\partial N_{i}^{e}}{\partial x} \frac{\partial N_{j}^{e}}{\partial x}\right) \mathrm{d} x \mathrm{~d} y .
\end{aligned}
$$

If the properties vary within the element, such as in the case of a non-linear analysis, the matrix given in Eq. (44) can be integrated as such to obtain the sub-matrix $K_{i, j}^{e}$ [13]. If the material 
properties are constant within the element, one has to integrate a sub-matrix

$$
\left[\begin{array}{c:c}
W_{i, j}^{x, x, e} & \vdots \\
\hdashline \ldots i, \ldots, y \\
\hdashline W_{i, j}^{x, x, e} & W_{i, j}^{x, y, e}
\end{array}\right],
$$

where

$$
W_{i, j}^{\phi, \psi, e}=t P_{i, j}^{\phi, \psi, e}=t \int_{e} \frac{\partial N_{i}^{e}}{\partial \phi} \frac{\partial N_{j}^{e}}{\partial \psi} \mathrm{d} x \mathrm{~d} y, \quad \phi \psi=x, y .
$$

\subsection{Algorithm to compute element stiffness matrix $\left[K^{e}\right]$ :}

The integrals of the products of global derivatives viz. $W_{i, j}^{x, x, e}, W_{i, j}^{x, y, e}, W_{i, j}^{y, x, e}$ and $W_{i, j}^{y, y, e}$ are first stored in the matrix $\left[K^{e}\right]$ itself. Thus for the nodal pairs $i, j$ we have the sub-matrix

$$
\left[K_{i, j}^{e}\right]=\left[\begin{array}{c:c}
W_{i, j}^{x, x}, e & W_{i, j}^{x, y, e} \\
\hdashline \ldots, i, \ldots & \ldots \ldots \\
W_{i, j}^{y, x}, e & W_{i, j}^{y, y, e}
\end{array}\right] .
$$

By use of the pre-computed universal numeric arrays, we can compute $P_{i, j}^{x, x, e}, P_{i, j}^{x, y, e}$ and $P_{i, j}^{p, y, e}$ for $i$, $j=1,2, \ldots, N$ (where $N$ is the number of nodes per element $e$ ) and hence $W_{i, j}^{\phi, \psi}, e, \phi, \psi=x, y$. We must note that this analytical integration is much simpler than even the arithmetic of evaluating the product of global derivatives at an arbitrary Gauss quadrature point.

We know that input to the computation of element stiffness matrix are the integrals $W_{i, j}^{x, x, e}$, $W_{i, j}^{x, y, e}, W_{i, j}^{y, x, e}$ and $W_{i, j}^{y, y, e}$, and they are already stored in $\left[K_{i, j}^{e}\right]$ and $\left[K^{e}\right]$ stores all integrals of the product of global derivatives.

The computation of element stiffness matrix can now be taken up using the matrix $\left[K^{e}\right]$. We create temporary storage location $Q_{1,1}, Q_{1,2}, Q_{2,1}$ and $Q_{2,2}$ as

$$
\begin{array}{ll}
Q_{1,1}=K_{2 i-1,2 j-1}^{e}, & Q_{2,1}=K_{2 i, 2 j-1}^{e}, \\
Q_{1,2}=K_{2 i-1,2 j}^{e}, & Q_{2,2}=K_{2 i, 2 j}^{e},
\end{array}
$$

in the element stiffness matrix subroutine, and compute and store the entries of element stiffness matrix again in the same matrix $\left[K^{e}\right]$. A subroutine STIFF which performs this operation is listed in Fig. 6 [13].

\section{Conclusion}

We have constructed some precomputed analytical numeric arrays for a special type of linear convex quadrilateral elements to obtain the finite element matrices for the solutions of $2 \mathrm{D}$ linear partial differential equations. This has been employed for the finite element discretization of a linear two-dimensional polygon into a set of linear triangles and each of these triangles is further discretized into three special linear quadrilateral elements which give rise to same Jacobian 


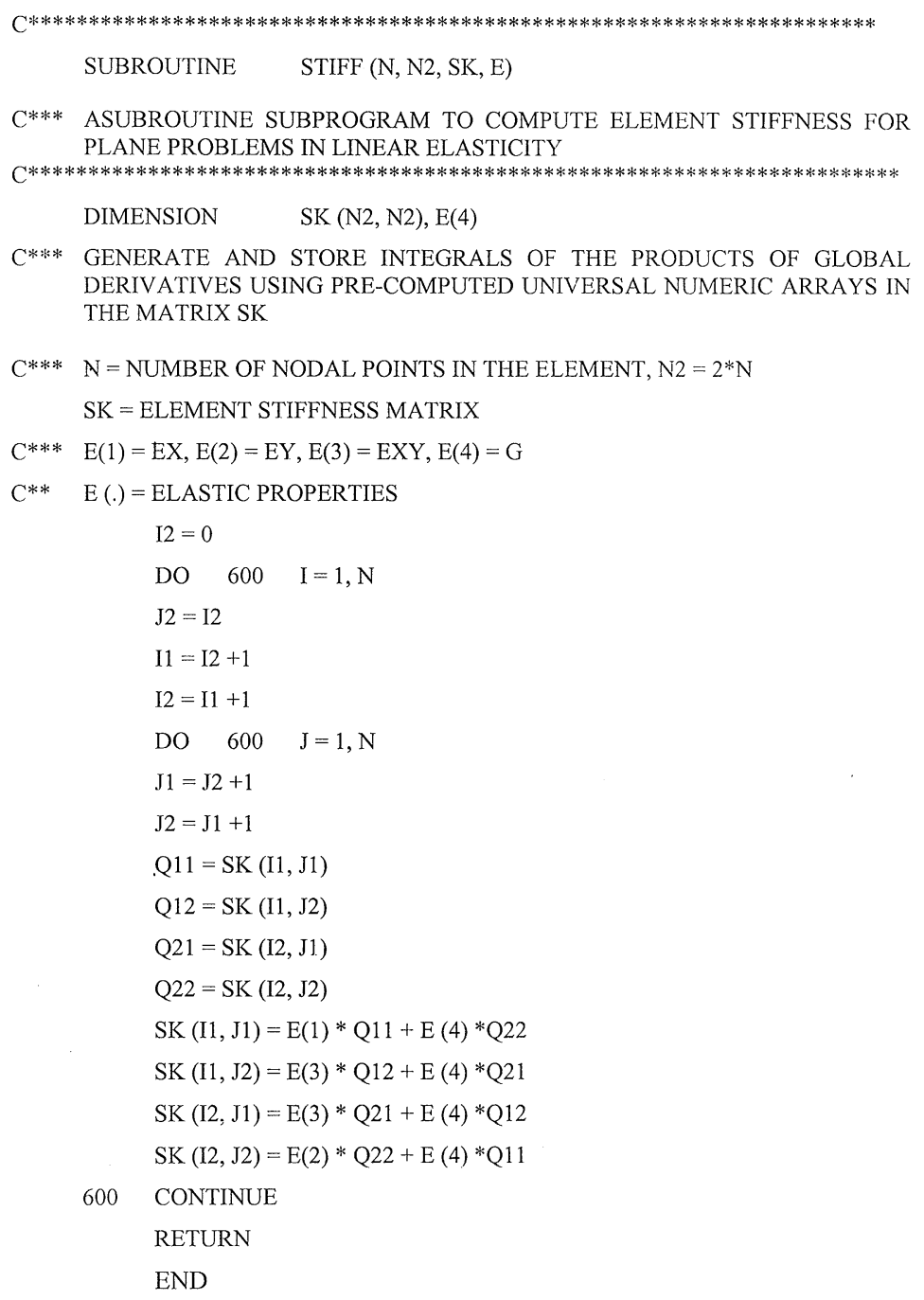

Fig. 6. FORTRAN subroutine for the calculation of the stiffness matrix.

expression viz. $(4+\xi+\eta)$ in local parametric $(\xi, \eta)$ coordinate systems. We have constructed these arrays only for the five commonly known quadrilateral element types that are used in FEM in a variety of disciplines. Exploiting these arrays may be inexpensive to solve a problem several times using highly refined meshes and/or higher order elements. We have then solved a torsion problem adaptively to demonstrate the effectiveness of the proposed computed arrays. In this regard we may notice that the Lagrangian elements are excellent compared to the standard serendipity elements. However, the concluding remark is that the proposed computed arrays can be modified easily also for the higher order elements if more accuracy is desired. 


\section{Acknowledgements}

The authors wish to thank the learned referee for his helpful comments on the paper which greatly enhanced the quality of presentation in this research work.

Appendix A. Explicit expressions of the coefficient matrices $\left[a_{\alpha, \beta}^{i, e}(t)\right]$ or $\left[a_{\alpha, \beta}^{j, e}(s)\right] ; t=s=x, y$

A.1. For Q8 (Fig. 4a) element $(i=1,2, \ldots, 8)$ of Eq. (31)

\begin{tabular}{|c|c|c|c|}
\hline $\begin{array}{l}a_{0,0}^{1, e}(t)=0, \\
a_{1, e}^{1, e}(t)=\left(-b_{t}+2 c_{t}\right) \\
a_{0,0}^{2, e}(t)=0, \\
a_{1,0}^{2, e}(t)=\left(b_{t}+2 c_{t}\right) \\
a_{0,0}^{3, e}(t)=0, \\
a_{1,0}^{3, e}(t)=\left(-b_{t}+2 c_{t}\right) \\
a_{0,0}^{4, e}(t)=0, \\
a_{1}^{4, e}(t)=\left(b_{t}+2 c_{t}\right) \\
a_{0,0}^{5, e}(t)=2 b_{t}, \\
\left.a_{1,0}^{5, e}(t)=-4 c_{t}\right) \\
a_{0,0}^{6, e}(t)=2 c_{t}, \\
\left.a_{1,0}^{6, e}(t)=2 d_{t}\right) \\
a_{0, e}^{7, e}(t)=-2 b_{t}, \\
a_{2, e}^{3, e}(t)=d_{t} \\
a_{0, e}^{8, e}(t)=-2 c_{t}, \\
\left.a_{1,0}^{8, e}(t)=-2 d_{t}\right)\end{array}$ & $\begin{array}{l}a_{0,1}^{1, e}(t)=\left(-2 b_{t}+c_{t}\right) \\
a_{2, e}^{1, e}(t)=\left(b_{t}+2 d_{t}\right) \\
a_{0, e}^{2, e}(t)=-\left(2 b_{t}+c_{t}\right) \\
a_{2, e}^{2, e}(t)=\left(b_{t}+2 d_{t}\right) \\
a_{0,1}^{3, e}(t)=\left(-2 b_{t}+c_{t}\right) \\
a_{2, e}^{3, e}(t)=\left(-b_{t}+2 d_{t}\right) \\
a_{0,1}^{4, e}(t)=-\left(2 b_{t}+c_{t}\right) \\
a_{2,0}^{4, e}(t)=\left(-b_{t}+2 d_{t}\right) \\
a_{0,1}^{5, e}(t)=2 d_{t}, \\
a_{2,0}^{5, e}(t)=-2\left(b_{t}+2 d_{t}\right) \\
a_{0,1}^{6, e}(t)=4 b_{t}, \\
a_{2, e}^{6, e}(t)=0 \\
a_{0,1}^{7, e}(t)=-2 d_{t}, \\
a_{2, e}^{7, e}(t)=2\left(b_{t}-2 d_{t}\right) \\
a_{0,1}^{8, e}(t)=4 b_{t}, \\
a_{2, e}^{8, e}(t)=0\end{array}$ & $\begin{array}{l}a_{1,1}^{1, e}(t)=2\left(b_{t}-c_{t}\right), \\
a_{0,2}^{1, e}(t)=-\left(c_{t}+2 d_{t}\right) \\
a_{1,1}^{2, e}(t)=-2\left(b_{t}-c_{t}\right), \\
a_{0,2}^{2, e}(t)=\left(c_{t}-2 d_{t}\right) \\
a_{1, e}^{3, e}(t)=2\left(-b_{t}+c_{t}\right), \\
a_{0,2}^{3, e}(t)=\left(c_{t}-2 d_{t}\right) \\
a_{1,1}^{4, e}(t)=2\left(b_{t}+c_{t}\right), \\
a_{0,2}^{4, e}(t)=-\left(c_{t}+2 d_{t}\right) \\
a_{1}^{5, e}(t)=4 c_{t}, \\
a_{0,2}^{5, e}(t)=0 \\
a_{1,1}^{6, e}(t)=4 b_{t}, \\
a_{0,2}^{6, e}(t)=2\left(-c_{t}+2 d_{t}\right) \\
a_{1}^{7, e}(t)=-4 c_{t}, \\
a_{0,2}^{7, e}(t)=0 \\
a_{1,2}^{8, e}(t)=-4 b_{t}, \\
a_{0,2}^{8, e}(t)=2\left(c_{t}+2 d_{t}\right)\end{array}$ & $\begin{array}{l}a_{2,1}^{1, e}(t)=-d_{t}, \\
a_{1,2}^{1, e}(t)=d_{t} \\
a_{2,1}^{2, e}(t)=-d_{t}, \\
a_{1,2}^{2, e}(t)=-d_{t} \\
a_{2,1}^{3, e}(t)=d_{t}, \\
a_{1,2}^{3, e}(t)=-d_{t} \\
a_{2,1}^{4, e}(t)=d_{t}, \\
a_{1,2}^{4, e}(t)=d_{t} \\
a_{2,1}^{5, e}(t)=2 d_{t}, \\
a_{1,2}^{5, e}(t)=0 \\
a_{2,1}^{6, e}(t)=0, \\
a_{1,2}^{6, e}(t)=2 d_{t} \\
a_{2,1}^{7, e}(t)=-2 d_{t} \\
a_{1,2}^{7, e}(t)=0 \\
a_{2,1}^{8, e}(t)=0, \\
a_{1,2}^{8, e}(t)=-2 d_{t}\end{array}$ \\
\hline
\end{tabular}

A.2. For $Q 9$ (Fig. 4a) element $(i=1,2, \ldots, 9)$ of Eq. (32)

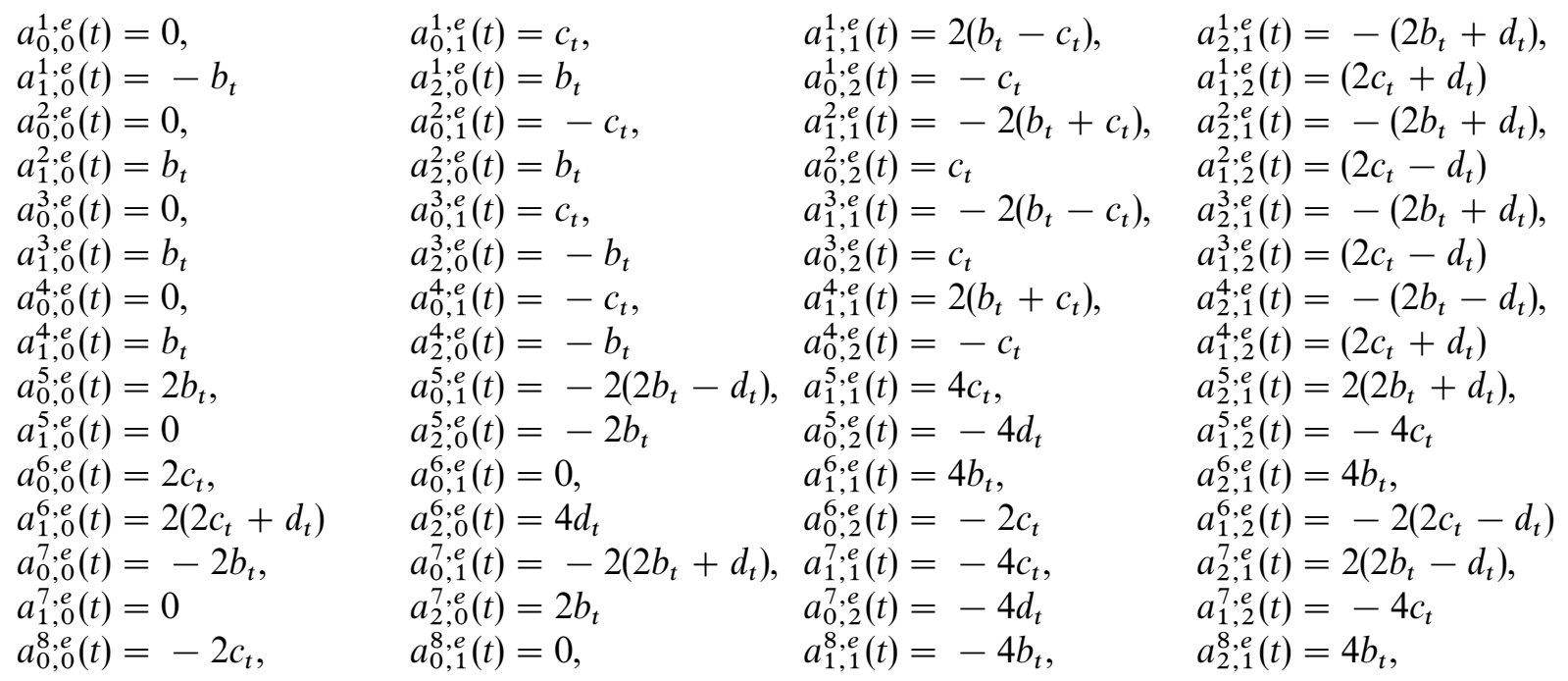




$\begin{array}{llll}a_{1,0}^{8, e}(t)=2\left(2 c_{t}-d_{t}\right) & a_{2,0}^{8, e}(t)=4 d_{t} & a_{0,2}^{8, e}(t)=2 c_{t} & \\ a_{0,0}^{9, e}(t)=0, & a_{0, e}^{9, e}(t)=8 b_{t}, & a_{1, e}^{9, e}(t)=0, & a_{2, e}^{9, e}(t)=-2\left(2 c_{t}+d_{t}\right) \\ a_{1,0}^{9, e}(t)=-8 c_{t} & a_{2,0}^{9, e}(t)=-8 d_{t} & a_{0,2}^{9, e}(t)=8 d_{t} & a_{1,2}^{9, e}(t)=8 c_{t}\end{array}$

A.3. For Q12 (Fig. 4b) element $(i=1,2, \ldots, 12)$ of Eq. (33)

$$
\begin{aligned}
& a_{0,0}^{1, e}(t)=10\left(-b_{t}+c_{t}\right), \quad a_{1,1}^{1, e}(t)=18\left(b_{t}-c_{t}\right), \\
& a_{1,0}^{1, e}(t)=2\left(5 b_{t}+9 c_{t}+5 d_{t}\right) \quad a_{0,2}^{1, e}(t)=9\left(3 b_{t}-c_{t}-2 d_{t}\right) \\
& a_{0,1}^{1, e}(t)=-2\left(9 b_{t}+5 c_{t}+5 d_{t}\right) \quad a_{2,1}^{1, e}(t)=9\left(3 c_{t}-d_{t}\right) \\
& a_{2,0}^{1, e}(t)=9\left(b_{t}-3 c_{t}+2 d_{t}\right) \\
& a_{0,0}^{2, e}(t)=-10\left(b_{t}+c_{t}\right) \text {, } \\
& a_{1,0}^{2, e}(t)=2\left(-5 b_{t}+9 c_{t}-5 d_{t}\right) \\
& a_{0,1}^{2, e}(t)=2\left(-9 b_{t}+5 c_{t}-5 d_{t}\right) \\
& a_{2,0}^{2, e}(t)=9\left(b_{t}+3 c_{t}+2 d_{t}\right) \\
& a_{0,0}^{3, e}(t)=10\left(b_{t}-c_{t}\right) \text {, } \\
& a_{1,2}^{1, e}(t)=9\left(-3 b_{t}+d_{t}\right) \\
& a_{1,1}^{2, e}(t)=-18\left(b_{t}+c_{t}\right) \text {, } \\
& a_{0,2}^{2, e}(t)=9\left(3 b_{t}+c_{t}-2 d_{t}\right) \\
& a_{2,1}^{2, e}(t)=-9\left(3 c_{t}+d_{t}\right) \\
& a_{1,2}^{2, e}(t)=9\left(3 b_{t}-d_{t}\right) \\
& a_{1,1}^{3, e}(t)=18\left(-b_{t}+c_{t}\right) \text {, } \\
& a_{1,0}^{3, e}(t)=2\left(5 b_{t}+9 c_{t}-5 d_{t}\right) \\
& a_{0,1}^{3, e}(t)=2\left(-9 b_{t}-5 c_{t}+5 d_{t}\right) \\
& a_{0,2}^{3, e}(t)=9\left(-3 b_{t}+c_{t}-2 d_{t}\right) \\
& a_{2,1}^{3, e}(t)=9\left(3 c_{t}+d_{t}\right) \\
& a_{2,0}^{3, e}(t)=9\left(-b_{t}+3 c_{t}+2 d_{t}\right) \\
& a_{0,0}^{4, e}(t)=10\left(b_{t}+c_{t}\right) \text {, } \\
& a_{1,0}^{4, e}(t)=2\left(-5 b_{t}+9 c_{t}+5 d_{t}\right) \\
& a_{0,1}^{4, e}(t)=2\left(-9 b_{t}+5 c_{t}+5 d_{t}\right) \\
& a_{2,0}^{4, e}(t)=9\left(-b_{t}-3 c_{t}+2 d_{t}\right) \\
& a_{1,2}^{3, e}(t)=-9\left(3 b_{t}+d_{t}\right) \\
& a_{1,1}^{4, e}(t)=18\left(b_{t}+c_{t}\right), \\
& a_{0,2}^{1, e}(t)=-9\left(3 b_{t}+c_{t}+2 d_{t}\right) \\
& a_{2,1}^{1, e}(t)=9\left(3 c_{t}+d_{t}\right) \\
& a_{1,2}^{1, e}(t)=9\left(3 b_{t}+d_{t}\right) \\
& a_{0,0}^{5, e}(t)=9\left(b_{t}-3 c_{t}\right), \\
& a_{1,0}^{5, e}(t)=-9\left(3 b_{t}+2 c_{t}+3 d_{t}\right) \\
& a_{0,1}^{5, e}(t)=9\left(3 c_{t}+d_{t}\right) \\
& a_{2,0}^{5, e}(t)=9\left(-b_{t}+9 c_{t}-2 d_{t}\right) \\
& a_{1,1}^{5, e}(t)=18 c_{t}, \\
& a_{0,2}^{5, e}(t)=0 \\
& a_{2,1}^{5, e}(t)=9\left(-9 c_{t}+d_{t}\right) \\
& a_{1,2}^{5, e}(t)=0 \\
& a_{3,0}^{1, e}(t)=-9\left(b_{t}+3 d_{t}\right), \\
& a_{0,3}^{1, e}(t)=9\left(c_{t}+3 d_{t}\right) \\
& a_{3,1}^{1, e}(t)=18 d_{t} \\
& a_{1,3}^{1, e}(t)=-18 d_{t} \\
& a_{3,0}^{2, e}(t)=9\left(b_{t}+3 d_{t}\right), \\
& a_{0,3}^{2, e}(t)=9\left(-c_{t}+3 d_{t}\right) \\
& \left.a_{3,1}^{2, e}(t)=-18 d_{t}\right) \\
& a_{1,3}^{2, e}(t)=18 d_{t} \\
& a_{3,0}^{3, e}(t)=9\left(-b_{t}+3 d_{t}\right) \text {, } \\
& a_{0,3}^{3, e}(t)=9\left(c_{t}-3 d_{t}\right) \\
& a_{3,1}^{3, e}(t)=18 d_{t} \\
& a_{1,3}^{3, e}(t)=-18 d_{t} \\
& a_{3,0}^{1, e}(t)=9\left(b_{t}-3 d_{t}\right) \text {, } \\
& a_{0,3}^{1, e}(t)=-9\left(c_{t}+3 d_{t}\right) \\
& a_{3,1}^{1, e}(t)=-18 d_{t} \\
& a_{1,3}^{1, e}(t)=18 d_{t} \\
& a_{3,0}^{5, e}(t)=27\left(b_{t}+3 d_{t}\right), \\
& a_{0,3}^{5, e}(t)=0 \\
& a_{3,1}^{5, e}(t)=-54 d_{t} \\
& a_{1,3}^{5, e}(t)=0 \\
& a_{0,0}^{6, e}(t)=9\left(b_{t}+3 c_{t}\right), \\
& a_{1,0}^{6, e}(t)=9\left(3 b_{t}-2 c_{t}+3 d_{t}\right) \\
& a_{0,1}^{6, e}(t)=9\left(-3 c_{t}+d_{t}\right) \\
& a_{2,0}^{6, e}(t)=9\left(b_{t}+9 c_{t}+2 d_{t}\right) \\
& a_{1,1}^{6, e}(t)=18 c_{t}, \\
& a_{0,2}^{6, e}(t)=0 \\
& a_{2,1}^{6, e}(t)=9\left(9 c_{t}+d_{t}\right) \\
& a_{1,2}^{6, e}(t)=0 \\
& a_{3,0}^{6, e}(t)=-27\left(b_{t}+3 d_{t}\right), \\
& a_{0,3}^{6, e}(t)=0 \\
& a_{3,1}^{6, e}(t)=54 d_{t} \\
& a_{1,3}^{6, e}(t)=0 \\
& a_{0,0}^{7, e}(t)=9\left(3 b_{t}+c_{t}\right), \\
& a_{1,0}^{7, e}(t)=9\left(3 b_{t}+d_{t}\right) \\
& a_{0,1}^{7, e}(t)=9\left(2 b_{t}-3 c_{t}+3 d_{t}\right) \\
& a_{2,0}^{7, e}(t)=0 \\
& a_{1,1}^{7, e}(t)=18 b_{t}, \\
& a_{0,2}^{7, e}(t)=9\left(-9 b_{t}-c_{t}+2 d_{t}\right) \\
& a_{2,1}^{7, e}(t)=0 \\
& a_{1,2}^{7, e}(t)=9\left(-9 b_{t}+d_{t}\right) \\
& a_{3,0}^{7, e}(t)=0 \text {, } \\
& a_{0,3}^{7, e}(t)=27\left(c_{t}-3 d_{t}\right) \\
& a_{3,1}^{7, e}(t)=0 \\
& a_{1,3}^{7, e}(t)=-54 d_{t} \\
& a_{0,0}^{8, e}(t)=9\left(-3 b_{t}+c_{t}\right), \\
& a_{1,1}^{8, e}(t)=18 b_{t}, \\
& a_{3,0}^{8, e}(t)=0 \text {, } \\
& a_{1,0}^{8, e}(t)=9\left(-3 b_{t}+d_{t}\right) \\
& a_{0,2}^{8, e}(t)=9\left(9 b_{t}-c_{t}+2 d_{t}\right) \text {, } \\
& a_{0,3}^{8, e}(t)=27\left(-c_{t}+3 d_{t}\right)
\end{aligned}
$$




$$
\begin{aligned}
& a_{0,1}^{8, e}(t)=9\left(2 b_{t}+3 c_{t}-3 d_{t}\right) \quad a_{2,1}^{8, e}(t)=0 \quad a_{3,1}^{8, e}(t)=0
\end{aligned}
$$

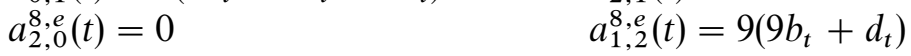

$$
\begin{aligned}
& a_{0,0}^{9, e}(t)=9\left(-b_{t}+3 c_{t}\right) \text {, } \\
& a_{1,0}^{9, e}(t)=9\left(-3 b_{t}-2 c_{t}+3 d_{t}\right) \\
& a_{1,1}^{9, e}(t)=-18 c_{t}, \\
& a_{0,2}^{9, e}(t)=0 \\
& a_{2,1}^{9, e}(t)=-9\left(9 c_{t}+d_{t}\right) \\
& a_{1,2}^{9, e}(t)=0 \\
& \begin{array}{l}
a_{0,1}^{9, e}(t)=9\left(3 c_{t}-d_{t}\right) \\
a_{2,0}^{9, e}(t)=9\left(b_{t}-9 c_{t}-2 d_{t}\right)
\end{array} \\
& a_{1,1}^{10, e}(t)=-18 c_{t}, \\
& a_{0,0}^{10, e}(t)=-9\left(b_{t}+3 c_{t}\right), \\
& a_{1,0}^{10, e}(t)=9\left(3 b_{t}-2 c_{t}-3 d_{t}\right) \\
& a_{0,1}^{10, e}(t)=-9\left(3 c_{t}+d_{t}\right) \\
& a_{2,0}^{10, e}(t)=9\left(2 b_{t}+9 c_{t}-2 d_{t}\right) \\
& a_{0,2}^{10, e}(t)=0 \\
& a_{2,1}^{10, e}(t)=9\left(9 c_{t}-d_{t}\right) \\
& a_{1,2}^{10, e}(t)=0 \\
& a_{0,0}^{11, e}(t)=-9\left(3 b_{t}+c_{t}\right), \\
& a_{1,0}^{11, e}(t)=9\left(3 b_{t}-d_{t}\right) \\
& a_{0,1}^{11, e}(t)=9\left(2 b_{t}-3 c_{t}-3 d_{t}\right) \\
& a_{2,0}^{11, e}(t)=0 \\
& a_{0,0}^{12, e}(t)=9\left(3 b_{t}-c_{t}\right), \\
& a_{1,0}^{12, e}(t)=-9\left(3 b_{t}+d_{t}\right) \\
& a_{0,1}^{12, e}(t)=9\left(2 b_{t}+3 c_{t}+3 d_{t}\right) \\
& a_{2,0}^{12, e}(t)=0 \\
& a_{0,2}^{11, e}(t)=9\left(9 b_{t}+c_{t}+2 d_{t}\right) \\
& a_{2,1}^{11, e}(t)=0 \\
& a_{1,2}^{1, e}(t)=-9\left(9 b_{t}+d_{t}\right) \\
& a_{1,1}^{12, e}(t)=-18 b_{t}, \\
& a_{0,2}^{12, e}(t)=9\left(-9 b_{t}+c_{t}+2 d_{t}\right) \\
& a_{2,1}^{12, e}(t)=0 \\
& a_{1,2}^{12, e}(t)=9\left(9 b_{t}-d_{t}\right)
\end{aligned}
$$

A.4. For Q16 (Fig. 4b) element $(i=1,2, \ldots, 16)$ of Eq. (34)

$$
\begin{aligned}
& a_{0,0}^{1, e}(t)=\left(-b_{t}+c_{t}\right), \\
& a_{1,0}^{1, e}(t)=-\left(b_{t}+18 c_{t}+d_{t}\right) \\
& a_{0,1}^{1, e}(t)=\left(18 b_{t}+c_{t}+d_{t}\right) \\
& a_{2,0}^{1, e}(t)=9\left(-b_{t}+3 c_{t}-2 d_{t}\right) \\
& a_{1,1}^{1, e}(t)=18\left(-b_{t}+c_{t}\right) \\
& a_{0,0}^{2, e}(t)=\left(b_{t}+c_{t}\right), \\
& a_{1,0}^{2, e}(t)=\left(b_{t}-18 c_{t}+d_{t}\right) \\
& a_{0,1}^{2, e}(t)=\left(18 b_{t}-c_{t}+d_{t}\right) \\
& a_{2,0}^{2, e}(t)=-9\left(b_{t}+3 c_{t}+2 d_{t}\right) \\
& a_{1,1}^{2, e}(t)=18\left(b_{t}+c_{t}\right) \\
& a_{0,2}^{1, e}(t)=9\left(-3 b_{t}+c_{t}+2 d_{t}\right), \\
& a_{2,1}^{1, e}(t)=9\left(-18 b_{t}-3 c_{t}+d_{t}\right) \\
& a_{1,2}^{1, e}(t)=9\left(3 b_{t}+18 c_{t}-d_{t}\right) \\
& a_{3,0}^{1, e}(t)=9\left(b_{t}+3 d_{t}\right) \\
& a_{0,3}^{1, e}(t)=-9\left(c_{t}+3 d_{t}\right) \\
& a_{0,2}^{2, e}(t)=9\left(-3 b_{t}-c_{t}+2 d_{t}\right), \quad a_{3,1}^{2, e}(t)=18\left(-9 b_{t}+d_{t}\right), \\
& a_{2,1}^{2, e}(t)=9\left(-18 b_{t}+3 c_{t}+d_{t}\right) \quad a_{1,3}^{2, e}(t)=-18\left(9 c_{t}+d_{t}\right) \\
& a_{1,2}^{2, e}(t)=9\left(-3 b_{t}+18 c_{t}+d_{t}\right) \quad a_{2,2}^{2, e}(t)=243\left(b_{t}+c_{t}\right) \\
& a_{3,0}^{2, e}(t)=9\left(b_{t}+3 d_{t}\right) \\
& a_{0,3}^{2, e}(t)=9\left(c_{t}-3 d_{t}\right) \\
& a_{0,0}^{3, e}(t)=\left(-b_{t}+c_{t}\right), \\
& a_{1,0}^{3, e}(t)=\left(-b_{t}-18 c_{t}+d_{t}\right) \\
& a_{0,1}^{3, e}(t)=\left(18 b_{t}+c_{t}-d_{t}\right) \\
& a_{2,0}^{3, e}(t)=9\left(b_{t}-3 c_{t}-2 d_{t}\right) \\
& a_{1,1}^{3, e}(t)=18\left(b_{t}-c_{t}\right) \\
& a_{0,2}^{3, e}(t)=9\left(3 b_{t}-c_{t}-2 d_{t}\right), \\
& a_{2,1}^{3, e}(t)=-9\left(18 b_{t}+3 c_{t}+d_{t}\right) \\
& a_{1,2}^{3, e}(t)=9\left(3 b_{t}+18 c_{t}+d_{t}\right) \\
& a_{3,0}^{3, e}(t)=9\left(b_{t}-3 d_{t}\right) \\
& a_{0,3}^{3, e}(t)=9\left(c_{t}-3 d_{t}\right) \\
& a_{3,2}^{2, e}(t)=81\left(3 b_{t}+d_{t}\right) \\
& a_{2,3}^{2, e}(t)=81\left(-3 c_{t}+d_{t}\right) \\
& a_{0,0}^{4, e}(t)=-\left(b_{t}+c_{t}\right), \\
& a_{1,0}^{4, e}(t)=\left(b_{t}-18 c_{t}+d_{t}\right) \\
& a_{0,2}^{4, e}(t)=9\left(3 b_{t}+c_{t}+2 d_{t}\right), \\
& a_{3,1}^{3, e}(t)=-18\left(9 b_{t}+d_{t}\right), \\
& a_{1,3}^{3, e}(t)=18\left(9 c_{t}+d_{t}\right) \\
& a_{2,2}^{3, e}(t)=243\left(-b_{t}+c_{t}\right) \\
& a_{3,2}^{3, e}(t)=81\left(3 b_{t}+d_{t}\right) \\
& a_{2,3}^{3, e}(t)=81\left(-3 c_{t}+d_{t}\right) \\
& a_{3,1}^{4, e}(t)=18\left(9 b_{t}+d_{t}\right), \\
& a_{2,1}^{4, e}(t)=9\left(-18 b_{t}+3 c_{t}-d_{t}\right) \\
& a_{1,3}^{4, e}(t)=18\left(9 c_{t}+d_{t}\right)
\end{aligned}
$$




$$
\begin{aligned}
& a_{0,1}^{4, e}(t)=\left(18 b_{t}-c_{t}-d_{t}\right) \\
& a_{2,0}^{4, e}(t)=9\left(b_{t}+3 c_{t}-2 d_{t}\right) \\
& a_{1,1}^{4, e}(t)=-18\left(b_{t}+c_{t}\right) \\
& a_{1,2}^{4, e}(t)=9\left(-3 b_{t}+18 c_{t}-d_{t}\right) \\
& a_{3,0}^{4, e}(t)=9\left(-b_{t}+3 d_{t}\right) \\
& a_{0,3}^{4, e}(t)=9\left(c_{t}+3 d_{t}\right) \\
& a_{0,0}^{5, e}(t)=9\left(-b_{t}+3 c_{t}\right), \\
& a_{1,0}^{5, e}(t)=9\left(3 b_{t}+2 c_{t}+3 d_{t}\right) \\
& a_{0,1}^{5, e}(t)=-9\left(18 b_{t}+3 c_{t}+d_{t}\right) \\
& a_{2,0}^{5, e}(t)=9\left(b_{t}-9 c_{t}+2 d_{t}\right) \\
& a_{1,1}^{5, e}(t)=18\left(27 b_{t}-c_{t}\right) \\
& a_{0,0}^{6, e}(t)=-9\left(b_{t}+3 c_{t}\right), \\
& a_{1,0}^{6, e}(t)=9\left(-3 b_{t}+2 c_{t}-3 d_{t}\right) \\
& a_{0,1}^{6, e}(t)=9\left(-18 b_{t}+3 c_{t}-d_{t}\right) \\
& a_{2,0}^{6, e}(t)=9\left(b_{t}+9 c_{t}+2 d_{t}\right) \\
& a_{1,1}^{6, e}(t)=-18\left(27 b_{t}+c_{t}\right) \\
& a_{0,0}^{7, e}(t)=-9\left(3 b_{t}+c_{t}\right), \\
& a_{1,0}^{7, e}(t)=9\left(-3 b_{t}+18 c_{t}-d_{t}\right) \\
& a_{0,1}^{7, e}(t)=9\left(-2 b_{t}+3 c_{t}-3 d_{t}\right) \\
& a_{2,0}^{7, e}(t)=81\left(3 b_{t}+3 c_{t}+2 d_{t}\right) \\
& a_{1,1}^{7, e}(t)=-18\left(b_{t}+27 c_{t}\right) \\
& a_{0,0}^{8, e}(t)=9\left(3 b_{t}-c_{t}\right), \\
& a_{1,0}^{8, e}(t)=9\left(3 b_{t}+18 c_{t}-d_{t}\right) \\
& a_{0,1}^{8, e}(t)=9\left(-2 b_{t}-3 c_{t}+3 d_{t}\right) \\
& a_{2,0}^{8, e}(t)=81\left(-3 b_{t}+3 c_{t}+2 d_{t}\right) \\
& a_{1,1}^{8, e}(t)=18\left(-b_{t}+27 c_{t}\right) \\
& a_{0,0}^{9, e}(t)=9\left(b_{t}-3 c_{t}\right), \\
& a_{1,0}^{9, e}(t)=9\left(3 b_{t}+2 c_{t}-3 d_{t}\right) \\
& a_{0,1}^{9, e}(t)=9\left(-18 b_{t}-3 c_{t}+d_{t}\right) \\
& a_{2,0}^{9, e}(t)=9\left(-b_{t}+9 c_{t}+2 d_{t}\right) \\
& a_{1,1}^{9, e}(t)=18\left(-27 b_{t}+c_{t}\right) \\
& a_{0,0}^{10, e}(t)=9\left(b_{t}+3 c_{t}\right), \\
& a_{1,0}^{10, e}(t)=9\left(-3 b_{t}+2 c_{t}+3 d_{t}\right) \\
& a_{0,1}^{10, e}(t)=9\left(-18 b_{t}+3 c_{t}+d_{t}\right) \\
& a_{2,0}^{10, e}(t)=9\left(-b_{t}-9 c_{t}+2 d_{t}\right) \\
& a_{1,1}^{10, e}(t)=18\left(27 b_{t}+c_{t}\right) \\
& a_{0,0}^{11, e}(t)=9\left(3 b_{t}+c_{t}\right), \\
& a_{1,0}^{11, e}(t)=9\left(-3 b_{t}+18 c_{t}+d_{t}\right) \\
& a_{0,1}^{11, e}(t)=9\left(-2 b_{t}+3 c_{t}+3 d_{t}\right) \\
& \begin{array}{l}
a_{0,2}^{5, e}(t)=81\left(3 b_{t}-3 c_{t}-2 d_{t}\right) \\
a_{2,1}^{5, e}(t)=9\left(18 b_{t}+9 c_{t}-d_{t}\right) \\
a_{1,2}^{5, e}(t)=81\left(-9 b_{t}-2 c_{t}+3 d_{t}\right) \\
a_{3, e}^{5, e}(t)=-27\left(b_{t}+3 d_{t}\right) \\
a_{0,3}^{5, e}(t)=243\left(c_{t}+d_{t}\right)
\end{array} \\
& a_{0,2}^{6, e}(t)=81\left(3 b_{t}+3 c_{t}-2 d_{t}\right), \\
& a_{2,1}^{6, e}(t)=9\left(18 b_{t}-9 c_{t}-d_{t}\right) \\
& a_{1,2}^{6, e}(t)=81\left(9 b_{t}-2 c_{t}-3 d_{t}\right) \\
& a_{3,0}^{6, e}(t)=27\left(b_{t}+3 d_{t}\right) \\
& a_{0,3}^{6, e}(t)=243\left(-c_{t}+d_{t}\right) \\
& a_{0,2}^{7, e}(t)=9\left(9 b_{t}+c_{t}-2 d_{t}\right), \\
& a_{2,1}^{7, e}(t)=81\left(2 b_{t}-9 c_{t}-3 d_{t}\right) \\
& a_{1,2}^{7, e}(t)=9\left(9 b_{t}-18 c_{t}-d_{t}\right) \\
& a_{3,0}^{7, e}(t)=243\left(b_{t}+d_{t}\right) \\
& a_{0,3}^{7, e}(t)=27\left(-c_{t}+3 d_{t}\right) \\
& a_{0,2}^{8, e}(t)=9\left(-9 b_{t}+c_{t}-2 d_{t}\right), \\
& a_{2,1}^{8, e}(t)=81\left(2 b_{t}+9 c_{t}+3 d_{t}\right) \\
& a_{1,2}^{8, e}(t)=-9\left(9 b_{t}+18 c_{t}+d_{t}\right) \\
& a_{3,0}^{8, e}(t)=243\left(-b_{t}+d_{t}\right) \\
& a_{0,3}^{8, e}(t)=27\left(c_{t}-3 d_{t}\right) \\
& a_{0,2}^{9, e}(t)=81\left(-3 b_{t}+3 c_{t}-2 d_{t}\right), \\
& a_{2,1}^{9, e}(t)=9\left(18 b_{t}+9 c_{t}+d_{t}\right) \\
& a_{1,2}^{9, e}(t)=-81\left(9 b_{t}+2 c_{t}+3 d_{t}\right) \\
& a_{3,0}^{9, e}(t)=27\left(-b_{t}+3 d_{t}\right) \\
& a_{0,3}^{9, e}(t)=243\left(c_{t}-d_{t}\right) \\
& a_{0,2}^{10, e}(t)=-81\left(3 b_{t}+3 c_{t}+2 d_{t}\right), \\
& a_{2,1}^{10, e}(t)=9\left(18 b_{t}-9 c_{t}+d_{t}\right) \\
& a_{1,2}^{10, e}(t)=81\left(9 b_{t}-2 c_{t}+3 d_{t}\right) \\
& a_{3,0}^{10, e}(t)=27\left(b_{t}-3 d_{t}\right) \\
& a_{0,3}^{10, e}(t)=-243\left(c_{t}+d_{t}\right) \\
& a_{0,2}^{11, e}(t)=-9\left(9 b_{t}+c_{t}+2 d_{t}\right), \\
& a_{2,1}^{11, e}(t)=81\left(2 b_{t}-9 c_{t}+3 d_{t}\right) \\
& a_{1,2}^{11, e}(t)=9\left(9 b_{t}-18 c_{t}+d_{t}\right) \\
& \begin{array}{l}
a_{2,2}^{4, e}(t)=-243\left(b_{t}+c_{t}\right) \\
a_{3,2}^{4, e}(t)=81\left(-3 b_{t}+d_{t}\right) \\
a_{2,3}^{4, e}(t)=81\left(3 c_{t}-d_{t}\right)
\end{array} \\
& a_{3,1}^{5, e}(t)=54\left(-9 b_{t}+d_{t}\right), \\
& a_{1,3}^{5, e}(t)=162\left(c_{t}-3 d_{t}\right) \\
& a_{2,2}^{5, e}(t)=243\left(-b_{t}+3 c_{t}\right) \\
& a_{3,2}^{5, e}(t)=243\left(3 b_{t}+d_{t}\right) \\
& a_{2,3}^{5, e}(t)=-81\left(9 c_{t}+d_{t}\right) \\
& a_{3,1}^{6, e}(t)=54\left(9 b_{t}-d_{t}\right), \\
& a_{1,3}^{6, e}(t)=162\left(c_{t}+3 d_{t}\right) \\
& a_{2,2}^{6, e}(t)=-243\left(b_{t}+3 c_{t}\right) \\
& a_{3,2}^{6, e}(t)=-243\left(3 b_{t}+d_{t}\right) \\
& a_{2,3}^{6, e}(t)=81\left(9 c_{t}-d_{t}\right) \\
& a_{3,1}^{7, e}(t)=162\left(b_{t}-3 d_{t}\right), \\
& a_{1,3}^{7, e}(t)=54\left(9 c_{t}+d_{t}\right) \\
& a_{2,2}^{7, e}(t)=-243\left(3 b_{t}+c_{t}\right) \\
& a_{3,2}^{7, e}(t)=-81\left(9 b_{t}+d_{t}\right) \\
& a_{2,3}^{7, e}(t)=243\left(3 c_{t}-d_{t}\right) \\
& a_{3,1}^{8, e}(t)=162\left(b_{t}+3 d_{t}\right), \\
& a_{1,3}^{8, e}(t)=-54\left(9 c_{t}+d_{t}\right) \\
& a_{2,2}^{8, e}(t)=-243\left(3 b_{t}-c_{t}\right) \\
& a_{3,2}^{8, e}(t)=81\left(9 b_{t}-d_{t}\right) \\
& a_{2,3}^{8, e}(t)=243\left(-3 c_{t}+d_{t}\right) \\
& a_{3,1}^{9, e}(t)=54\left(9 b_{t}+d_{t}\right), \\
& a_{1,3}^{9, e}(t)=-162\left(c_{t}+3 d_{t}\right) \\
& a_{2,2}^{9, e}(t)=243\left(b_{t}-3 c_{t}\right) \\
& a_{3,2}^{9, e}(t)=243\left(3 b_{t}-d_{t}\right) \\
& a_{2,3}^{9, e}(t)=81\left(-9 c_{t}+d_{t}\right)
\end{aligned}
$$




$$
\begin{aligned}
& a_{2,0}^{11, e}(t)=81\left(-3 b_{t}-3 c_{t}+2 d_{t}\right) \quad a_{3,0}^{11, e}(t)=243\left(b_{t}-d_{t}\right) \\
& a_{1,1}^{11, e}(t)=18\left(b_{t}+27 c_{t}\right) \\
& a_{0,3}^{11, e}(t)=-27\left(c_{t}+3 d_{t}\right) \\
& a_{3,2}^{11, e}(t)=81\left(-9 b_{t}+d_{t}\right) \\
& a_{2,3}^{11, e}(t)=243\left(3 c_{t}-d_{t}\right) \\
& a_{0,0}^{12, e}(t)=9\left(-3 b_{t}+c_{t}\right), \\
& a_{1,0}^{12, e}(t)=9\left(3 b_{t}+18 c_{t}+d_{t}\right) \\
& a_{0,2}^{12, e}(t)=9\left(9 b_{t}-c_{t}-2 d_{t}\right), \\
& a_{3,1}^{12, e}(t)=162\left(-b_{t}+3 d_{t}\right), \\
& a_{2,1}^{12, e}(t)=81\left(2 b_{t}+9 c_{t}-3 d_{t}\right) \\
& a_{1,3}^{12, e}(t)=54\left(9 c_{t}-d_{t}\right) \\
& a_{0,1}^{12, e}(t)=-9\left(2 b_{t}+3 c_{t}+3 d_{t}\right) \\
& a_{1,2}^{12, e}(t)=9\left(-9 b_{t}-18 c_{t}+d_{t}\right) \\
& a_{2,2}^{12, e}(t)=243\left(-3 b_{t}+c_{t}\right) \\
& a_{2,0}^{12, e}(t)=81\left(3 b_{t}-3 c_{t}+2 d_{t}\right) \\
& a_{3,0}^{12, e}(t)=-243\left(b_{t}+d_{t}\right) \\
& a_{1,1}^{12, e}(t)=18\left(b_{t}-27 c_{t}\right) \\
& a_{0,3}^{12, e}(t)=27\left(c_{t}+3 d_{t}\right) \\
& a_{3,2}^{12, e}(t)=81\left(9 b_{t}+d_{t}\right) \\
& a_{2,3}^{12, e}(t)=-243\left(3 c_{t}+d_{t}\right) \\
& a_{0,0}^{13, e}(t)=243\left(b_{t}-c_{t}\right), \\
& a_{0,2}^{13, e}(t)=81\left(-9 b_{t}+3 c_{t}+2 d_{t}\right), \\
& a_{3,1}^{13, e}(t)=486\left(b_{t}-3 d_{t}\right), \\
& a_{2,1}^{13, e}(t)=81\left(-2 b_{t}-27 c_{t}+3 d_{t}\right) \\
& a_{1,3}^{13, e}(t)=486\left(-c_{t}+3 d_{t}\right) \\
& a_{1,2}^{13, e}(t)=81\left(27 b_{t}+2 c_{t}-3 d_{t}\right) \\
& a_{2,2}^{13, e}(t)=729\left(b_{t}-c_{t}\right) \\
& a_{3,0}^{13, e}(t)=729\left(b_{t}+d_{t}\right) \\
& a_{0,3}^{13, e}(t)=-729\left(c_{t}+d_{t}\right) \\
& a_{3,2}^{13, e}(t)=-243\left(9 b_{t}+d_{t}\right) \\
& a_{2,3}^{13, e}(t)=243\left(9 c_{t}+d_{t}\right) \\
& a_{0,0}^{14, e}(t)=243\left(b_{t}+c_{t}\right), \\
& a_{1,0}^{14, e}(t)=81\left(9 b_{t}-2 c_{t}+3 d_{t}\right) \\
& a_{0,1}^{14, e}(t)=81\left(2 b_{t}-9 c_{t}+3 d_{t}\right) \\
& a_{2,0}^{14, e}(t)=-81\left(3 b_{t}+9 c_{t}+2 d_{t}\right) \\
& a_{1,1}^{14, e}(t)=486\left(b_{t}+c_{t}\right) \\
& a_{0,2}^{14, e}(t)=81\left(-9 b_{t}-3 c_{t}+2 d_{t}\right), \\
& a_{3,1}^{14, e}(t)=486\left(-b_{t}+3 d_{t}\right), \\
& a_{2,1}^{14, e}(t)=81\left(-2 b_{t}+27 c_{t}+3 d_{t}\right) \\
& a_{1,3}^{14, e}(t)=-486\left(c_{t}+3 d_{t}\right) \\
& a_{1,2}^{14, e}(t)=81\left(-27 b_{t}+2 c_{t}+3 d_{t}\right) \\
& a_{2,2}^{14, e}(t)=729\left(b_{t}+c_{t}\right) \\
& a_{3,0}^{14, e}(t)=-729\left(b_{t}+d_{t}\right) \\
& a_{3,2}^{14, e}(t)=243\left(9 b_{t}+d_{t}\right) \\
& a_{0,3}^{14, e}(t)=-729\left(c_{t}+d_{t}\right) \\
& a_{2,3}^{14, e}(t)=243\left(-9 c_{t}+d_{t}\right) \\
& a_{0,0}^{15, e}(t)=243\left(-b_{t}+c_{t}\right), \\
& a_{1,0}^{15, e}(t)=81\left(-9 b_{t}-2 c_{t}+3 d_{t}\right) \\
& a_{0,2}^{15, e}(t)=81\left(9 b_{t}-3 c_{t}+2 d_{t}\right), \\
& a_{3,1}^{15, e}(t)=-486\left(b_{t}+3 d_{t}\right), \\
& a_{2,1}^{15, e}(t)=-81\left(2 b_{t}+27 c_{t}+3 d_{t}\right) \\
& a_{1,3}^{15, e}(t)=486\left(c_{t}+3 d_{t}\right) \\
& a_{0,1}^{15, e}(t)=81\left(2 b_{t}+9 c_{t}-3 d_{t}\right) \\
& a_{1,2}^{15, e}(t)=81\left(27 b_{t}+2 c_{t}+3 d_{t}\right) \\
& a_{2,2}^{15, e}(t)=729\left(-b_{t}+c_{t}\right) \\
& a_{2,0}^{15, e}(t)=81\left(3 b_{t}-9 c_{t}-2 d_{t}\right) \\
& a_{1,1}^{15, e}(t)=486\left(b_{t}-c_{t}\right) \\
& a_{3,0}^{15, e}(t)=729\left(b_{t}-d_{t}\right) \\
& a_{0,3}^{15, e}(t)=729\left(-c_{t}+d_{t}\right) \\
& a_{3,2}^{15, e}(t)=243\left(-9 b_{t}+d_{t}\right) \\
& a_{2,3}^{15, e}(t)=243\left(9 c_{t}-d_{t}\right) \\
& a_{0,0}^{16, e}(t)=-243\left(b_{t}+c_{t}\right), \\
& a_{1,0}^{16, e}(t)=81\left(9 b_{t}-2 c_{t}-3 d_{t}\right) \\
& a_{0,1}^{16, e}(t)=81\left(2 b_{t}-9 c_{t}-3 d_{t}\right) \\
& a_{2,0}^{16, e}(t)=81\left(3 b_{t}+9 c_{t}-2 d_{t}\right) \\
& a_{0,2}^{16, e}(t)=81\left(9 b_{t}+3 c_{t}+2 d_{t}\right), \\
& a_{3,1}^{16, e}(t)=486\left(b_{t}+3 d_{t}\right), \\
& a_{2,1}^{16, e}(t)=81\left(-2 b_{t}+27 c_{t}-3 d_{t}\right) \\
& a_{1,3}^{16, e}(t)=486\left(c_{t}-3 d_{t}\right) \\
& a_{1,2}^{16, e}(t)=81\left(-27 b_{t}+2 c_{t}-3 d_{t}\right) \\
& a_{2,2}^{16, e}(t)=-729\left(b_{t}+c_{t}\right) \\
& a_{3,0}^{16, e}(t)=729\left(-b_{t}+d_{t}\right) \\
& a_{1,1}^{16, e}(t)=-486\left(b_{t}+c_{t}\right) \\
& a_{0,3}^{16, e}(t)=729\left(c_{t}+d_{t}\right) \\
& a_{3,2}^{16, e}(t)=243\left(9 b_{t}-d_{t}\right) \\
& a_{2,3}^{16, e}(t)=-243\left(9 c_{t}+d_{t}\right)
\end{aligned}
$$


Appendix B. The precomputed universal numeric symmetric arrays $[S]_{\mathrm{n} \times \mathrm{n}}, n=3,8,12,15$ defined in Eqs. (32)-(34)

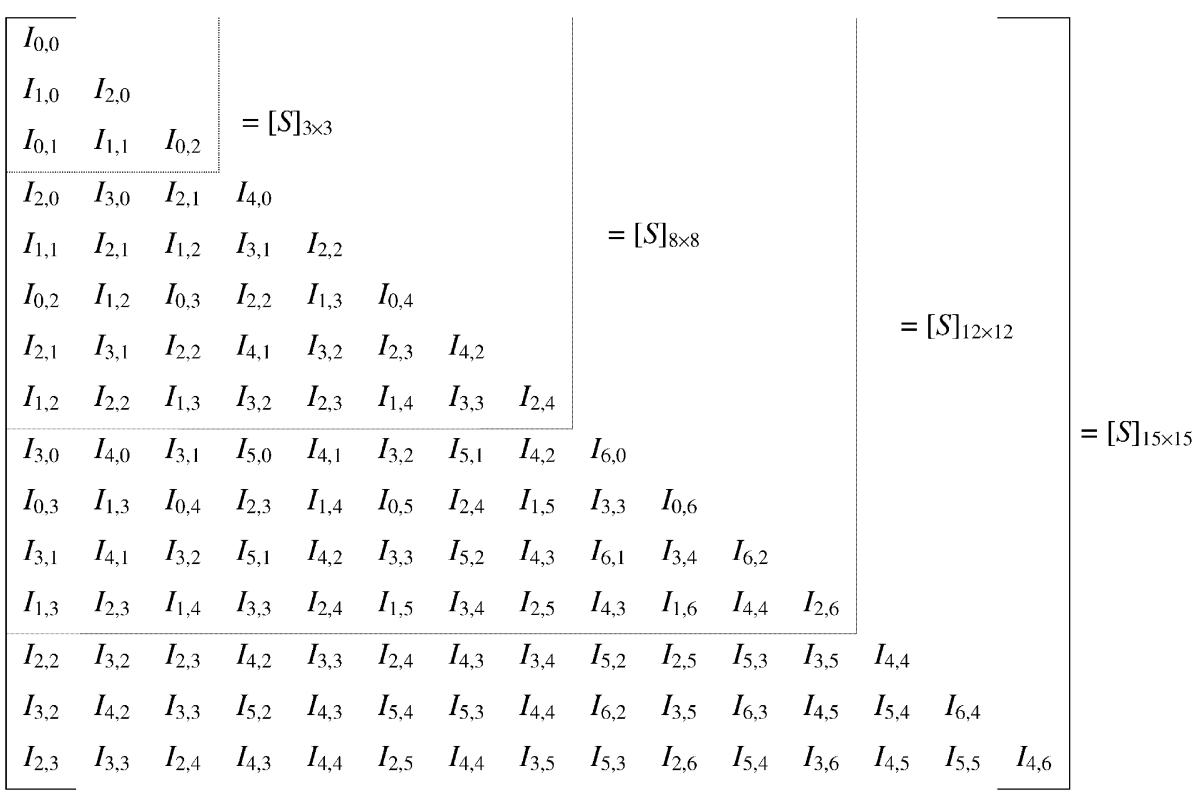

where

$I_{0,0}=1.046496288$,

$$
I_{5,0}=-0.040394717
$$$$
I_{4,3}=-0.012193999,
$$

$I_{1,0}=-0.092992575$,

$I_{4,1}=-0.020120150$

$I_{6,2}=0.052606226$,

$I_{2,0}=0.355592309$,

$I_{3,2}=-0.019843708$,

$I_{5,3}=0.004396426$,

$I_{1,1}=0.016377991$,

$I_{6,0}=0.154376098$,

$I_{4,4}=0.044379572$,

$I_{3,0}=-0.056279920$,

$I_{5,1}=0.007202769$,

$I_{6,3}=-0.008759143$,

$I_{2,1}=-0.032755982$,

$I_{4,2}=0.073277832$,

$I_{5,4}=-0.008759143$,

$I_{4,0}=0.215128717$,

$I_{3,3}=0.006097999$,

$I_{6,4}=0.031866020$,

$I_{3,1}=0.009990965$,

$I_{5,1}=-0.014560413$,

$I_{5,5}=0.003170553$,

$I_{2,2}=0.121032965$,

$I_{5,2}=-0.014250663$,

$N . B: I_{m, n}=I_{n, m}$ for all concerned integral values of $m, n$.

\section{References}

[1] O.C. Zienkiewicz, The Finite Element Method, 3rd Edition, Mc-Graw Hill, New York, 1977.

[2] K.J. Bathe, Finite Element Procedures, Prntice-Hall, Englewood Cliffs, NJ, 1996.

[3] G. Yogawa, G.W. Ye, S. Yoshimura, A numerical integration scheme for finite element method based on symbolic manipulation, Int. J. Numer. Methods Eng. 29 (1990) 1539-1549.

[4] M. Okabe, Analytical integral formula related to convex quadrilateral finite elements, Comput. Methods Appl. Mech. Eng. 29 (1981) 201-218. 
[5] D.K. Babu, G.F. Pinder, Analytical integration formulae for linear isoparametric finite elements, Int. J. Numer. Methods Eng. 20 (1984) 1153-1166.

[6] A. Mizukami, Some integration formulas for a four node isoparametric element, Comput. Methods Appl. Mech. Eng. 59 (1986) 111-121.

[7] H.T. Rathod, Some analytical integration formulae for a four node isoparametric element, Comput. Struct. 30 (1988) 1101-1109.

[8] K.E. Barrett, Explicit eight-noded quadrilateral elements, Finite Element. Anal. Des. 31 (1999) 209-222.

[9] M.P. Rossow, I.N. Katz, Hierarchical finite elements and pre-computed arrays, Int. J. Numer. Methods Eng. 12 (1978) 977-999.

[10] S.H. Nguyen, An accurate finite element formulation for linear elastic torsion calculations, Comput. Struct. 42 (1992) 707-711.

[11] S.P. Timoshenko, J.N. Goodier, Theory of Elasticity, Mc-Graw Hill, New York, 1970.

[12] R.J. Roark, Formulas for Stress and Strain, Mc-Graw Hill, New York, 1965.

[13] A.K. Gupta, Efficient numerical integration of element stiffness matrices, Int. J. Numer. Methods Eng. 19 (1983) $1410-1413$. 